Vol. 6, n² | 2002

Varia

\title{
Le Code criminel canadien, 1892-1939 : Le contrôle des armes à feu
}

\section{Gérald Pelletier}

\section{(2) OpenEdition \\ 12 Journals}

Édition électronique

URL : https://journals.openedition.org/chs/414

DOI : $10.4000 /$ chs. 414

ISSN : 1663-4837

Éditeur

Librairie Droz

\section{Édition imprimée}

Date de publication : 1 décembre 2002

Pagination : $51-79$

ISBN : 2-600-00812-8

ISSN : $1422-0857$

\section{Référence électronique}

Gérald Pelletier, «Le Code criminel canadien, 1892-1939 : Le contrôle des armes à feu », Crime, Histoire \& Sociétés / Crime, History \& Societies [En ligne], Vol. 6, n² | 2002, mis en ligne le 25 février 2009, consulté le 23 mars 2022. URL : http://journals.openedition.org/chs/414; DOI : https://doi.org/ $10.4000 /$ chs 414

Ce document a été généré automatiquement le 23 mars 2022.

(c) Droz 


\title{
Le Code criminel canadien, 1892-1939 : Le contrôle des armes à feu
}

\author{
Gérald Pelletier
}

1 L'objectif de cet article est d'étudier le contrôle des armes à feu dans le droit pénal canadien - en y incluant, de façon plus large, les armes blanches et à air comprimé - en prenant comme point de départ de la recherche l'état de la loi telle qu'exprimée lors de l'édiction du Code criminel canadien en 1892. Le terme de l'étude est l'année 1939 alors que le Canada entre en guerre contre l'Allemagne.

2 D'entrée de jeu, il convient d'exposer les trois particularités que présente le Code criminel canadien. Sa première caractérisque est de comprendre non seulement une partie substantive regroupant les actes interdits et les peines correspondantes (comme c'est le cas en Europe continentale et en Amérique latine) mais également une partie procédurale. Or, et c'est la deuxième particularité, ces deux parties ne sont pas cloisonnées en ce sens que l'on retrouve très souvent certains éléments de procédure à l'intérieur même de la partie substantive. La dernière caractéristique du Code canadien est d'être en quelque sorte " ouvert ", c'est-à-dire que tout amendement adopté par le Parlement à l'une de ses dispositions y est immédiatement incorporé, au lieu de simplement indiquer la loi modifiant l'article dans une note infrapaginale ${ }^{2}$.

3 Créé donc en 1892, le Code criminel canadien traversera le premier demi-siècle de son histoire sous le regard attentif du législateur qui n'hésite pas à en remodeler souvent le contenu. À preuve, environ le quart de ses articles auront déjà été amendés en $1927^{3}$. Dans le seul champ des armes à feu, entre 1892 et 1939, le Parlement canadien adoptera, à l'intérieur de 11 lois, pas moins de 35 amendements qui viennent modifier 13 articles de la partie substantive du Code criminel. Ces retouches à répétition qui n'ont évidemment rien de fortuit méritent d'être appréhendées car elles laissent facilement deviner que la question du contrôle des armes à feu est très sensible aux aléas de la conjoncture. L'exemple contemporain du rôle joué par la tuerie à l'École 
polytechnique de l'Université de Montréal, en 1989, dans la relance du débat sur les armes à feu au Canada illustre bien notre propos ${ }^{4}$.

4 Nous entendons donc précisément décoder ici les nombreuses résonances sociales liées à une activité législative aussi fébrile en démontrant que les transformations subies par le Code criminel canadien en matière d'armes à feu sont l'expression d'une réaction à l'évolution de la conjoncture du pays. Nous verrons qu'en période d'exacerbation des tensions causées tantôt par le climat politique (national ou international), tantôt par la grogne sociale née des ratées de l'économie ou de l'inégalité de ses retombées, les autorités gouvernementales adoptent invariablement le réflexe de criminaliser davantage la question des armes à feu en gardant tout particulièrement dans leur mire les nouveaux arrivants et, de façon plus large, tous ceux qui ne sont pas encore naturalisés Canadiens.

5 Des changements profonds ont façonné la jeune nation canadienne entre les années 1892 et 1939. Tout au long de cette période en effet, les traits de l'horizon démographique, économique, politique et social du pays ont été en mutation constante.

6 La fluidité de la configuration du paysage démographique s'explique par l'accroissement spectaculaire de la population canadienne qui s'enclenche au tournant du siècle. Le phénomène est déterminant: un peu plus de 5 millions de personnes habitaient le Canada en 1901; en l'espace de vingt ans seulement, ce nombre est grimpé à près de 9 millions. Ces moments de croissance démographique intense, que permet une économie qui progresse alors à un rythme effréné, sont le fruit d'un courant migratoire au débit soutenu. Un exemple tiré de la seule communauté ukrainienne : en 1891 arrivent au Canada les deux premiers immigrants ukrainiens; en 1914, ils sont déjà plus de 150000 installés dans l'Ouest canadien sur une large bande de terre s'étirant de Winnipeg à Edmonton ${ }^{5}$ ! Cette arrivée massive de cohortes d'immigrants s'inscrit vraiment dans la réalité canadienne après 1896 , alors que le gouvernement adapte sa politique d'immigration pour qu'elle s'harmonise avec trois grands changements survenus sur la scène internationale : les États-Unis n'ont presque plus de terres à bon marché à offrir aux immigrants; le prix du blé est à la hausse et, à l'inverse, les frais de transport maritime des marchandises sont à la baisse ${ }^{6}$. Une conjoncture internationale que Clifford Sifton, ministre responsable, entre autres, de la question de l'immigration au sein de l'équipe libérale du Premier ministre Wilfrid Laurier (1896-1911), saura mettre à profit en recrutant ouvertement à l'étranger des fermiers compétents pour peupler les Prairies. Et pour la première fois, le réservoir traditionnel de l'immigration s'enrichira de ressortissants de l'Europe de l'Est: des Allemands, des Autrichiens, des Slaves..., bref des gens de langues et de cultures différentes.

7 Ce contexte de santé démographique exceptionnelle que traduit d'ailleurs la création dès 1905 de deux nouvelles provinces, l'Alberta et la Saskatchewan, a comme corollaire la prospérité économique fulgurante que connaît alors le pays. La dynamique est la suivante : l'essor économique du pays sert d'abord de bougie d'allumage au mouvement migratoire et s'en nourrit lui-même ensuite (main-d'œuvre, marché intérieur, etc.). En gros, la multiplication par quatre des investissements étrangers entre 1901 et 1921 conjuguée à la fois à une hausse des prix sur le marché international, à partir de l'automne 1896, et à un accroissement de la demande du marché de la Grande-Bretagne 
et de l'Europe en produits agricoles (blé, farine, viande, produits laitiers), concourent à déclencher le boom de l'Ouest et favorisent, du même coup, le développement des industries manufacturières du Québec et de l'Ontario. Ces deux provinces centrales, déjà bien engagées sur la voie de l'industrialisation au début du siècle, récolteront, d'ailleurs, la majorité des nouveaux investissements industriels et hydro-électriques de la période, si bien que profitant beaucoup, par surcroît, de la demande suscitée ensuite par la guerre, elles continueront à naviguer dans les années 1920 sur les eaux de la croissance économique. Par opposition à l'Ontario et au Québec qui sont le cœur industriel et urbain du pays, les provinces de l'Ouest pourtant responsables du miracle économique de l'avant-guerre par leurs exportations de blé (Manitoba, Saskatchewan et Alberta), ont néanmoins été négligées en termes d'investissements industriels, si bien qu'au lendemain de la guerre, elles vogueront, elles, sur des eaux plus instables Ces retombées inégales de la croissance économique ne seront pas sans créer un ressentiment certain chez ceux qui s'en sentent lésés. De façon générale toutefois, c'est bien campée sur les rails de la prospérité que la majeure partie de la société canadienne s'engage dans le virage de la dépression économique des années 1930 : la sortie de piste, on le sait, sera catastrophique! Il faudra en fait attendre l'éclatement de la Seconde Guerre mondiale pour, ironiquement, échapper à la dérive socio-économique de ces années de misère.

8 Sur la scène politique, c'est sous le règne de l'équipe libérale de Wilfrid Laurier qu'à partir du tournant du siècle se produit l'effervescence économique qui fait entrer le pays d'un océan à l'autre dans l'ère industrielle et urbaine. L'arrivée du gouvernement Laurier représente en soi un événement, puisqu'elle met fin à la domination à peu près ininterrompue des Conservateurs sur l'arène politique depuis le début de la Confédération. Aux performances économiques et au renouveau politique associés à l'ère de Laurier, il faut malheureusement ajouter aussi les tensions ethniques nées de l'éveil du nationalisme canadien-anglais issu des guerres impériales. Le militarisme canadien-anglais provoqué par la guerre des Boers (1899-1902) se solde, en effet, par l'éclosion d'un climat de suspicion inter-ethnique - les francophones du Québec s'opposent catégoriquement à l'envoi de troupes en Afrique du Sud ${ }^{8}$ - que viendra raviver plus tard l'éclatement de la Première Guerre mondiale. Dans le contexte de la guerre de 1914, les immigrants originaires de pays alors en guerre avec l'Angleterre et le Canada essuieront l'hostilité de certains éléments de la population canadienne qui, atteints par la fièvre martiale, pèchent par excès de patriotisme; leur hostilité sera d'ailleurs exploitée par le Gouvernement conservateur de Borden (1911-1917) qui dépouillera de leur droit de vote les ressortissants originaires de pays ennemis et arrivés au Canada depuis 1902. L'adoption d'une loi dite "Loi des élections en temps de guerre » fait en réalité partie de la stratégie électorale du nouveau parti unioniste - une alliance de Libéraux et de Conservateurs dirigée par le même Borden - qui prendra alors le pouvoir (1917-1920)9. Mentionnons enfin que, toujours au plan politique, la misère engendrée par la crise économique des années 1930 convie le gouvernement canadien à plus d'interventionnisme dans la vie des citoyens.

Sur le plan social, les phénomènes de l'industrialisation et de l'immigration massive se traduiront par un formidable mouvement d'urbanisation et par l'émergence, au fil de la conjoncture, de tensions entre les ethnies et les classes. L'explosion urbaine marquera la fin de l'ère de l'environnement à prédominance rurale dans lequel vivaient encore $60 \%$ des Canadiens en 1901. Au Québec et en Ontario, plus de gens vivent à la ville qu'à la campagne dès 1911, fruit de l'immigration et de l'exode des ruraux. L'Ouest agricole 
du pays qui attire tant d'immigrants en quête d'une vie meilleure, vit aussi à l'heure de la frénésie urbaine, comme en témoigne alors la création des villes de Calgary et d'Edmonton en Alberta, et celles de Regina et de Saskatoon en Saskatchewan. De 4398 personnes en 1901, la population de Calgary grimpera ainsi à 43704 en 1911 et à 63305 en $1921^{10}$. L'environnement urbain dans lequel vit un Canadien sur deux dès $1921^{11}$ est caractérisé entre autres par la montée de la classe ouvrière et par les nombreuses inégalités sociales charriées par une économie dont l'industrie et la finance sont devenues les maîtres mots. Car le foisonnement des problèmes sociaux se fait au rythme de la croissance des villes et les ghettos urbains dans lesquels s'entassent tout particulièrement les nouveaux travailleurs immigrants, spécialement ceux qui sont marqués par le stigmate de l'ethnicité, incarnent avec le plus d'éclat l'envers social de ce boom économique. C'est d'ailleurs, de façon plus large, en filigrane à l'implantation du capitalisme industriel que s'est organisée, au cours des années 1880-1929, la classe ouvrière canadienne ${ }^{12}$. Le militantisme de ces travailleurs dont les effectifs grimpent en flèche pendant la Première Guerre mondiale ne sera pas sans inquiéter les autorités gouvernementales, incapables de résister à la tentation d'y voir un lien avec la révolution bolchevique et la montée du communisme.

10 C'est donc sur cette trame historique que le Code criminel canadien vivra son premier demi-siècle d'existence et que s'échafaudera la structure du contrôle des armes à feu.

\section{1. - Les armes offensives et la législation criminelle en 1892}

Dans le Code criminel canadien, la question des armes offensives ${ }^{13}$ est traitée dans la section des Crimes contre l'ordre public, une rubrique dans laquelle se retrouvent aussi les infractions liées à la trahison, aux attroupements illégaux, aux substances explosives et aux séditions. En 1892, au moment où naît le Code criminel, un total de 16 articles (102-117) viennent spécifiquement baliser le port et la possession d'armes offensives. En cette fin de XIX ${ }^{e}$ siècle, le champ d'interdits délimité par le Code se voulait aussi large que possible, comme en font foi lesgloses de quelques-uns des articles qui y sont alors insérés : "Contrebandiers portant des armes offensives "; « Porter des couteaux à gaine dans les ports de mer»; "S'approcher armé d'une assemblée publique»; "Possession d'armes près de travaux publics "; "Porter un pistolet sans cause raisonnable »; « Vendre un pistolet ou un fusil à vent [c'est-à-dire à air comprimé] à un mineur "; «Vente d'armes dans les Territoires du Nord-Ouest », etc. Les codificateurs de 1892 voulaient donc ratisser large et certains des articles qu'ils couchent alors dans le Code résisteront bien au passage du temps, puisqu'on les retrouvera à peu près inchangés encore en 1954. D'autres, par contre, subiront les balafres répétées de nombreux amendements apportés dans le sillon de la conjoncture. Le contenu de ces articles dont le contour sera souvent ciselé par le législateur véhicule, pour l'essentiel, en 1892 , les interdits suivants.

D'abord, il y a prohibition du port d'armes. L'article 105 interdit en effet à quiconque de porter un pistolet ou un fusil à vent ailleurs que dans sa propre maison, boutique, magasin ou bureau d'affaires, à moins d'avoir préalablement obtenu un "certificat d'exemption ». La loi prévoit cependant des exceptions à l'obligation de détenir un tel certificat comme l'appartenance à un corps policier ou militaire et la crainte légitime d'un individu que sa personne, sa famille ou sa propriété ne soient en danger. L'âge 
minimum pour obtenir un certificat d'exemption est de seize ans et le juge de paix chargé de déterminer la pertinence des raisons formulées par le demandeur ne peut l'accorder que pour une période maximum de douze mois. Enfin, les contrevenants sont passibles, sur conviction par voie sommaire, d'une amende de $5 \$$ à $25 \$$ ou d'un emprisonnement d'un mois; cette peine augmente lorsqu'un individu est reconnu coupable de port illégal d'armes lors d'une arrestation (20\$ à $50 \$$ ou trois mois de prison) ${ }^{14}$. Pour souligner au passage l'importance de ces amendes par rapport à d'autres éléments du niveau de vie de l'époque, notons à titre d'exemple que cordonniers et tailleurs montréalais gagnaient à l'été 1887 un salaire hebdomadaire moyen de $8 \$^{15}$.

Le port d'armes blanches (poignard, dague, couteau à gaine...) est également proscrit et rend son auteur passible, après déclaration sommaire de culpabilité, d'une amende de $10 \$$ à $50 \$$ ou, à défaut de paiement, d'un emprisonnement maximum de 30 jours $^{16}$.

Le Code défend aussi de donner ou de vendre un pistolet ou un fusil à vent à un mineur âgé de moins de seize ans, sous peine de se voir infliger (conviction par voie sommaire) une amende pouvant aller jusqu'à $50 \$$.

Enfin, obligation est faite aux vendeurs de pistolets ou de fusils à vent de conserver un registre de leurs transactions dans lequel doivent être consignés la date de la vente, les noms de l'acheteur et du fabricant de l'arme, ainsi que tout autre détail pouvant servir à identifier ladite arme. Le non-respect de cette clause peut valoir une amende maximum de $25 \$^{17}$.

16 Soulignons, en terminant, que les questions de confiscation et de saisie sont régies par deux statuts dont les dispositions, sans avoir été intégrées formellement dans le Code criminel de 1892, en font néanmoins partie ${ }^{18}$.

17 Prohibition du port d'armes, criminalisation de l'aliénation d'armes aux mineurs et obligation pour le vendeur de conserver un registre de transactions, voilà donc en matière d'armes offensives trois traits du Code criminel de 1892 auxquels le gouvernement canadien apportera par la suite de nombreuses retouches. Et, signalonsle tout de suite, nulle part dans ce premier échafaudage codifié n'est dessinée la moindre équation entre le statut d'« étranger » et l'accès aux armes à feu.

\section{2. - La stigmatisation de l'« étranger » dans le Code criminel}

18 Entre 1892 et 1927, le Parlement du Canada adopte un total de 9 amendements pour remodeler certains articles du Code criminel traitant des armes offensives. Plus précisément, ces changements sont adoptés en 1909, 1913, 1919, 1920 et 1921.

19 C'est donc en 1909 (chapitre 9, article 2) que, depuis la codification, le législateur se penche pour la première fois sur la question des armes offensives. L'article 123 du Code crimine ${ }^{19}$ qui prohibe le port d'armes blanches et l'article 124 qui interdit spécifiquement - à moins d'y être obligé par son métier - de porter des couteaux à gaine dans les villes, voient les peines prévues s'alourdir: la sentence d'emprisonnement maximum, applicable jusque là seulement en cas de non-paiement de l'amende, sera dorénavant imposée seule ou en sus de l'amende et elle passera de 30 jours à 3 mois. En fait, la durée de l'enfermement carcéral pourra même grimper à 6 mois lorsque l'individu condamné à une peine jumelant amende et prison aura négligé d'acquitter ladite amende. Tant au Sénat qu'à la Chambre des Communes, cette 
pénalisation accrue est justifiée par l'utilisation devenue fréquente au Canada des armes blanches et notamment du couteau-poignard ${ }^{20}$. La peine se veut donc dissuasive pour enrayer une criminalité que l'on dit montante au pays. En réponse aux critiques d'un député (M. Monk) sur le réflexe de répression législative du gouvernement en matière pénale, le solliciteur général ${ }^{21}$ de l'époque, Jacques Bureau, rétorque :

«Si nous avons multiplié le nombre des infractions et essayé d'aggraver les peines, c'est dans l'effort tenté pour endiguer le flot toujours montant de la criminalité dans notre pays. Si je comprends bien le principe sur lequel repose le Code pénal, c'est celui-ci : il vous est défendu de faire tel acte et si vous le faites, vous êtes passible d'un châtiment $\|^{22}$.

En 1913, les forces policières du pays convainquent les autorités législatives canadiennes de resserrer de quelques crans le contrôle des armes en introduisant, entre autres, la notion de permis de port d'armes. Disparaissent donc de l'article $118 \mathrm{du}$ Code l'ancien « certificat d'exemption ${ }^{23}$ et l'exception qui l'accompagnait, relative à la crainte légitime d'un individu que sa personne, sa famille ou sa propriété ne soient en danger. Apparait à leur place l'interdiction de porter sans permis toute arme offensive (arme à feu, arme à air comprimé et «arme blanche») ${ }^{24}$ pouvant être dissimulée sur soi, ailleurs que dans sa propre maison, boutique, magasin ou bureau d'affaires. À cette restriction étendue du droit de porter des armes se greffe une pénalisation accrue : une amende maximum de $100 \$$ et/ou un emprisonnement de trois mois remplacent en effet l'amende maximum de $25 \$$ ou l'incarcération d'un mois que prévoyait jusque-là le Code. Cette peine est d'ailleurs celle prévue pour toutes les autres infractions visées par l'article 118. Car l'amendement du 6 juin 1913 (chapitre 46, article 4) dont il est ici question ne fait pas que prohiber le seul port d'armes. Il criminalise aussi le fait d'aliéner (vente, don, prêt) quelqu'arme offensive à un individu non détenteur d'un permis. Il réitère également, en l'étoffant, l'obligation pour le vendeur de conserver un registre de ses transactions (date de la vente, nom de l'acheteur, description de l'arme, date et lieu d'émission du permis, nom et fonction de la personne habilitée à émettre ce permis); une peine plus sévère sera donc désormais infligée au vendeur fautif ${ }^{25}$. Il contraint, de plus, les personnes dorénavant autorisées à émettre de tels permis - c'està-dire principalement les milieux policiers à la place des juges de paix - à en conserver un double et condamne le fait d'émettre un permis sans autorisation légitime. Il donne enfin le pouvoir à tout agent de la paix de fouiller toute personne qu'il soupçonne de porter une arme sans permis et de saisir ladite arme. Voilà pour l'article 118 du Code criminel. Dernière modification apportée en 1913 (chapitre 13, article 5) qui touche cette fois l'article 119 du Code, il est défendu d'aliéner quelqu'arme à feuà un mineur âgé de moins de seize ans et non pas seulement des pistolets ou des fusils à vent, tel que formulé jusqu'alors dans la loi ${ }^{26}$.

21 À quoi attribuer ce resserrement du contrôle des armes observable en 1909 et en 1913 ? Le ministre de la Justice lui-même, C. J. Doherty, déclare en termes à peine voilés à la Chambre des Communes qu'il l'attribue, pourrait-on dire, aux « habitudes culturelles » de certains immigrants, en faisant un lien on ne peut plus direct entre la hausse de la criminalité et la présence toujours plus massive de nouveaux arrivants :

«I think it is evident that we are coming to have a very large increase in the number of crimes of violence, which I think may be directly attributed to the general carrying of arms [...]. While our population is increasing, being fed by constant streams of immigration - in which, generally speaking, we rejoice - it is coming to contain, I think, and regrettably so, a larger proportion of persons with 
regard to whom it is unsafe and certainly undesirable that the procuring of weapons [...] should be as easy as it is under present conditions ${ }^{27}$.

Nous l'avons dit, le Canada vit depuis le tournant du siècle des moments de croissance démographique intense, fruit d'un déferlement migratoire soutenu. Pour tout dire, de forts contingents d'immigrants débarquent au pays à un rythme que ne peut tout simplement pas absorber la population canadienne déjà en place. C'est cette inquiétude générée par l'espace qu'occupent désormais les nouveaux arrivants dans le paysage démographique canadien qui est traduite dans le Code criminel en 1913 comme en 1909. Mais, et la nuance est importante, cette transposition se fait encore de façon non ségrégative en ce sens que même si les députés discourent sur la responsabilité des nouveaux venus dans la hausse de la criminalité, les modifications qu'ils apportent à certains articles du Code traitant des armes offensives s'appliquent alors à l'ensemble de la population, sans égard à l'ethnicité. Or, le déclenchement de la Première Guerre mondiale, la révolution bolchevique et l'affirmation du mouvement syndical sur la scène nationale entraîneront un durcissement marqué du Code et l'apparition d'un double registre d'interdits: l'un pour les sujets britanniques, l'autre pour les "étrangers ", c'est-à-dire les personnes non naturalisées britanniques, groupe dans lequel certains se remarquent d'autant plus aisément que, depuis le début du siècle, de fortes cohortes d'immigrants sont originaires de l'Europe de l'Est. Ainsi, en 1919, à la demande notamment du commissaire de police en chef du Canada et de la majorité des conseils municipaux de l'Ontario représentant des villes d'au moins 10000 habitants, le gouvernement adoptera un amendement qui ajoute à l'interdiction, depuis 1913, de porter des armes sans autorisation, la défense, pour les étrangers seulement, de posséder (donc même dans leur propre maison) quelqu'arme (" any weapon, air gun, device or contrivance aforesaid») sans s'être préalablement munis d'un permis ${ }^{28}$. Cet amendement crée donc une loi à deux vitesses: la possession d'armes est partout illégale pour un " étranger » qui n'a pas de permis; elle est par contre légale pour un sujet britannique à l'intérieur de sa propre maison, boutique, magasin ou bureau d'affaires, car seul le port d'armes à l'extérieur de ces lieux l'oblige depuis 1913 à acquérir un permis. L'idée de ce traitement différencié née du climat d'ultrapatriotisme et d'anti-communisme qui alors prévaut et rend l'« étranger » suspect, voire dangereux, ressort clairement des plaidoyers acheminés au ministère de la Justice par les instigateurs de changements au Code. Le Conseil municipal de Guelph (Ontario) adresse ainsi, le 5 février 1919, une résolution au Premier ministre Robert Borden l'enjoignant «to pass legislation providing that no alien be permitted to have in his possession any firearm or other deadly weapon [...] in view of the number of recent cases in which deadly weapons have been used as a means of settling disputes in which men of foreign birth were involved $»^{29}$. Cette demande d'épingler spécifiquement l'étranger dans le Code criminel pour juguler la hausse de la criminalité aura des échos favorables à la Chambre des Communes.

«Depuis deux ans - dira le solliciteur général Guthrie, un Libéral de l'équipe unioniste - presque tous les crimes les plus graves, meurtres et autres, qui ont été commis dans l'Ontario, l'ont été par des étrangers.

[...] Sur la totalité des détenus dans les pénitenciers et les prisons, il y a une proportion d'environ 75 pour cent d'étrangers. Je donnerai les chiffres pour la prison de Guelph, qui est la ville où j'habite. Le 22 avril dernier, comme l'a fait remarquer le juge en chef d'Ontario, nous avions dans la prison 13 détenus, tous étrangers, pas un seul sujet britannique ». 
à ces suggestions d'amendements qui stigmatisent les étrangers dans le dossier des armes à feu. Partant du principe que ce sont les étrangers qui, en très grande majorité au Canada, transportent des armes sur eux ${ }^{33}$, le ministre suppléant de la Justice, Arthur Meighen, un Conservateur, expose la pertinence des amendements proposés en ces termes :

« La grande difficulté à laquelle doit faire face la police actuellement est le nombre considérable de revolvers et d'autres armes que les gens gardent chez eux, prêts à s'en servir à n'importe quel moment, et qu'ils se passent de l'un à l'autre sans que les autorités civiles puissent exercer aucune surveillance [...].

Les autorités de la police [...] me disent que la seule manière de contrôler l'abus est d'obliger les étrangers à avoir un permis pour pouvoir garder des armes chez eux. [Ces hommes] ne sont pas des sujets britanniques; souvent ce sont des étrangers de nationalité ennemie $[. . .]^{34}$.

L'acquiescement du gouvernement canadien à ces demandes de réformes au Code criminel lui est alors d'autant moins difficile qu'elles s'arrimaient fort bien à sa propre vision des choses, et ce, pour plusieurs raisons. D'abord, il avait déjà créé en 1915, dans cette même rubrique des Crimes contre l'ordre public (sous-section : crimes de trahison et lèse-majesté), l'article $75 \mathrm{~A}$ qui criminalisait le fait d'aider un sujet de nationalité étrangère, dont la patrie d'origine était en guerre avec l'Angleterre, à quitter le Canada sans permission. Cet acte criminel rendait son auteur passible de deux ans d'emprisonnement «...à moins, précisait l'article, que la personne accusée puisse prouver qu'elle n'avait pas l'intention d'aider l'ennemi ». Soulignons au passage que le gouvernement, trop patriotiquement occupé à débusquer les traîtres ${ }^{35}$, ne se formalisa guère de l'accroc qu'il faisait ici au principe britannique de la présomption d'innocence $^{36}$. Pas plus qu'il ne se gêna ensuite, en 1917, pour retirer leur droit de vote - nous l'avons déjà dit - à tous les immigrants issus de pays «ennemis » qui étaient arrivés au Canada depuis 1902! Troisièmement, cette marginalisation - aux relents de xénophobie - des " étrangers " se retrouve encore dans la création, toujours en 1919, des deux articles 97A et 97B du Code criminel qui définissent avec une très grande fermeté les deux notions d'association illégale et d'activité séditieuse, les affectant d'une peine pouvant aller jusqu'à vingt ans de prison. Inspirés d'un Ordre en Consei ${ }^{37}$ du 28 septembre 1918 qui déclarait illégales quatorze associations de travailleurs et partis politiques généralement réunis derrière la bannière de l'ethnicité (Russian Social Democratic Party, Ukrainian Social Democratic Party, Chinese Labour Association, Industrial Workers of the World, etc.) et recommandés par un Comité spécial de la Chambre des Communes chargé de se pencher sur la législation afférente 
aux menées séditieuses, ces deux amendements sont adoptés dans la foulée de l'éclatement de grèves d'un bout à l'autre du pays. Ce mouvement de revendications des travailleurs culmine à Winnipeg (Manitoba) au printemps de 1919: la ville est paralysée et le dénouement du conflit nécessite l'application de la loi sur l'émeute. "The Borden government remained convinced that revolution was under way", écrivent les historiens Robert Craig Brown et Ramsay Cook $^{38}$. Ce mécontentement syndical qui gronde alors sur la scène nationale et qui témoigne de la montée croissante des forces ouvrières canadiennes en quête de meilleurs salaires et conditions de travail, s'exprime au moment même où les clameurs ouvrières de la révolution bolchevique sèment l'émoi sur la scène internationale. Dans sa lecture des événements, le gouvernement canadien sera prompt à lier les deux phénomènes ${ }^{39}$ et à soupçonner les "étrangers» de menées séditieuses: il réagira notamment en amendant le Code criminel. Ce que dit haut et fort le sénateur Lougheed sur la question traduit d'ailleurs assez bien l'état de nervosité législative du moment et le réflexe gouvernemental de faire de l'étranger un bouc émissaire :

«[...] durant ces derniers mois, principalement depuis la signature de l'armistice, l'attention du Gouvernement a été appelée sur l'organisation, à travers le Canada, d'un grand nombre d'associations formées surtout d'étrangers, dans le but de poursuivre une propagande qui conduirait non seulement au mépris de la loi, mais au renversement de notre forme actuelle de gouvernement. Outre ce qu'a révélé la grève de sympathie de Winnipeg, le gouvernement $a$ eu des preuves indéniables qu'un mouvement de cette nature se propageait par tout le Canada. Il est par conséquent fortement à désirer qu'une loi soit insérée dans nos statuts qui aura pour effet de supprimer ces sortes d'associations $»^{40}$.

27 Perçus comme des traîtres en puissance à cause de la Première Guerre mondiale, comme des révolutionnaires potentiels à cause des événements de Winnipeg et de la prise du pouvoir par les communistes en Russie, les étrangers que l'on avait déjà brimés dans leurs droits d'association et leur liberté d'accès à l'information ${ }^{41}$ et traités comme des citoyens de seconde classe sur la scène électorale, n'allaient certainement pas être traités avec plus de délicatesse dans le dossier des armes à feu. Non que l'opposition fit défaut : un député (Mr. Jacobs) clamera par exemple son désaccord en ces termes :

«Si c'est une bonne loi, pourquoi ne pas l'appliquer aux sujets britanniques aussi bien qu'aux étrangers? Pourquoi faire une distinction entre les étrangers et ceux qui ne le sont pas? [...]

[...] un sujet britannique peut faire autant de mal [avec un revolver] qu'un étranger, et personnellement, je ne considère pas que tous les sujets britanniques soient des modèles parfaits, ni que tous les étrangers soient des êtres dangereux. [...]. Le fait même qu'un homme ne se trouve pas être né en territoire britannique n'en fait pas nécessairement un être dangereux. Si cette loi doit être adoptée, elle devrait s'appliquer à toutes les classes de citoyens, sujets britanniques comme étrangers ».

Comme tout nouvel arrivant dans ce pays aspire un jour à la citoyenneté britannique, poursuit sarcastiquement le député, il faut donc en conclure qu'au moment même où il sera drapé de sa nouvelle citoyenneté, le même individu alors métamorphosé pourra dorénavant garder sans permis (et donc sans danger pour sa communauté) des armes dans sa maison. Ce à quoi acquiesce le ministre Meighen, comme allant de soi...42. Même réprobation de la part du député McMaster qui, s'il est plus enclin à entrebâiller la porte du «double standard » dans certains secteurs, tient néanmoins fermement à ce que tous soient traités sur le même pied (accès limité) en matière de contrôle des armes à feu : 
" Je ne saurais souscrire à l'idée qu'il doive exister une loi pour les étrangers et une autre loi pour les sujets britanniques. Il est évident, cela va sans dire, que lorsqu'il s'agit de voter ou d'exercer une fonction de l'État, il devrait y avoir une différence, mais en ce qui concerne les affaires de police ou de paix publique, il n'est pas judicieux [...] d'établir deux règles. J'approuve volontiers la proposition tendant à obliger ceux qui ont des armes meurtrières à demander un permis. Notre population étrangère a fait usage peut-être plus fréquemment de ces armes que les gens de sang ou de nationalité britannique, et parmi toutes les classes on devrait restreindre l'habitude de porter des armes sur soi ou de les tenir cachées à la maison. [...] et je demanderais au ministre d'étendre à tous les sujets l'obligation d'obtenir un permis qu'il veut imposer aux [seuls] étrangers. [...]

Je déclare que c'est une erreur d'avoir une politique qui est contraire aux traditions britanniques [à savoir] d'établir certains règlements de police pour des étrangers et d'autres pour les citoyens du pays. [...]

Pourquoi marquerions-nous d'une tache [c'est-à-dire stigmatiserions-nous] les étrangers respectables qui composent l'énorme majorité en mettant en vigueur des restrictions de police que vous leur appliquez et non à d'autres? $»^{43}$.

Or le gouvernement fera non seulement la sourde oreille à ces récriminations, mais le ministre Meighen en rajoutera en disant que cette politique du double standard à l'égard des étrangers s'inscrit dans la tradition juridique du pays :

"L'honorable député, réplique ainsi Meighen, croit que c'est antibritannique d'établir pareille distinction entre les étrangers et les sujets britanniques, mais il n'en est rien. Il n'y a presque pas de statuts où l'on ne fasse pareille distinction. D'après notre loi des terres fédérales [...] les étrangers n'ont aucun droit à une terre fédérale. [...] Ils sont sujets au bannissement tandis que les sujets britanniques ne le sont pas - et la distinction existe partout $»^{44}$.

En d'autres termes, la ségrégation du passé légitime celle du présent...

31 L'amendement du 7 juillet 1919 (chapitre 46, article 2) qui enlève plus de droits aux étrangers qu'au reste de la population en matière d'accès aux armes à feu, introduit d'autres changements à l'article $118 \mathrm{du}$ Code criminel. D'abord, la défense de porter cachées sur soi des armes dangereuses est élargie pour englober le transport d'armes dissimulées, par exemple, dans un sac. Une telle argumentation aurait été utilisée devant les tribunaux pour contourner la loi. Ensuite, les permis qui sont émis ne seront valables qu'à l'intérieur de la juridiction couverte par l'autorité habilitée à les délivrer. Enfin, à la demande du surintendant de l'Immigration, les agents d'immigration compteront désormais, à côté des forces policières et militaires, au rang des groupes qui peuvent porter des armes sans permis ${ }^{45}$. Compte tenu, nous venons de le voir, des perceptions gouvernementale et policière des immigrants, l'adoption de cette disposition ne surprend guère, pas plus, d'ailleurs, que le ton emprunté par le surintendant dans la formulation de sa demande d'amendement : «I think it is highly desirable that a slight amendment should be made which will authorize Immigration officers to carry weapons for self-defence. Several of our officers already carry revolvers and are practically obliged to do so $»^{46}$.

Soulignons en terminant que la législation criminelle du 7 juillet 1919, déjà très chargée par la portée et la sévérité des changements qu'elle introduit, crée encore (chapitre 46, article 3) un nouvel article au Code criminel : l'article 128A. Ses dispositions interdisent le port ou la décharge d'une arme à feu, sans autorisation, sur des propriétés du ministère de la Défense nationale et ce, sous peine d'une amende maximum de $100 \$$ ou/ et d'un emprisonnement pouvant aller jusqu'à 60 jours. Demandé par le sous-ministre 
dudit ministère, l'article 128A visait à l'évidence à convaincre les soldats de se départir de certaines habitudes contractées pendant la guerre... ${ }^{47}$.

La stigmatisation des étrangers à laquelle concourait la réglementation de la possession d'armes à feu de juillet 1919 sera suivie quelques mois plus tard (le 10 novembre 1919) d'un autre amendement à l'article 118 du Code criminel : la portée de la loi y sera élargie de manière à y inclure explicitement un plus grand nombre de sortes d'armes à feu : «being an alien, has in his possession any pistol, rifle, shot-gun, revolver, firearm or offensive weapon without having a permit therefor... $»^{48}$. Le pouvoir politique veut ainsi colmater toute brèche possible dans l'interprétation de la loi qui en rendrait la lettre non conforme à l'esprit. Disons-le tout de suite, le Code criminel canadien conservera jusqu'en 1950 des dispositions empreintes de xénophobie dans ce dossier, en interdisant aux seuls étrangers la possession de quelqu'arme à feu sans avoir préalablement obtenu un permis.

La seule exception à ce scénario et encore n'est-elle pas entière - se produit le $1^{\mathrm{er}}$ juillet 1920 (chapitre 43, article 2) alors que le gouvernement généralise pour tous les Canadiens l'obligation de détenir un permis pour posséder une arme à feu et la porter. N'en sont exemptés que les sujets britanniques déjà propriétaires d'un fusil de chasse (et non pas - la distinction est importante - ceux qui possèdent déjà une carabine, considérée comme plus dangereuse). La disposition élargit en plus la portée de la loi en y incluant des éléments tels les canons et les mitrailleuses ${ }^{49}$. L'amendement durcit donc la loi de façon significative puisque, dans le cas des sujets britanniques, l'interdit qui s'appliquait jusque là au seul port d'armes qu'on pouvait cacher sur sa personne s'étend désormais (à une exception près) à la possession de toute arme à feu. La sévérité nouvelle de cette législation, qui a certainement le mérite d'être non ségrégative dans son application, vise à exercer un contrôle plus strict du commerce des armes. Le ministre de la Justice et procureur général, Charles Joseph Doherty, un Conservateur dans le gouvernement unioniste de Borden, l'explique en ces termes :

«Il a été jugé utile, dans les circonstances actuelles, de réglementer le commerce des armes à feu en général. Il ressort des rapports faits par la gendarmerie et, en outre, dans une certaine mesure, d'informations transmises aux autorités militaires, que dans certaines localités, à diverses périodes, il a existé [...] une activité insolite dans l'acquisition d'armes à feu. [...]

[...] Le but est de nous permettre d'obtenir la connaissance du trafic des armes à feu dangereuses et de surveiller les personnes qui les ont en leur possession. Les rapports de police ont insisté [...] sur la grande importance de cette connaissance et de cette surveillance $»^{50}$.

Mais pourquoi donc? Parce que les autorités en place restent alors hantées par la possibilité d'une menace insurrectionnelle alimentée par l'idéologie communiste, une attitude qualifiée d'obsessionnelle par le député Power :

«[...] parce que le ministre de la Justice ou le gouvernement se sont mis dans la tête pour une raison ou pour une autre, que le bolchevisme ou toute autre doctrine en " isme " se propage en Canada, ils veulent forcer aussitôt tous les citoyens à se procurer des permis pour avoir le droit de garder des armes à feu dans leurs maisons $»^{51}$.

Pressé par un des opposants au projet de loi (le sénateur Watson) d'expliquer pourquoi la possession antérieure d'une carabine par un sujet britannique nécessitera un permis et non pas celle d'un fusil de chasse, le sénateur Sir James Lougheed répond en des termes qui ne laissent aucun doute sur la nature politique de l'amendement proposé : 
«[...] si l'on était menacé d'émeute ou d'insurrection, dit-il, on pourrait importer un grand nombre de carabines. Et si tout sujet britannique avait le droit d'avoir une carabine sans permission, l'accumulation de toutes ces carabines pourrait précipiter beaucoup de troubles et de difficultés ». en soustrayant les sujets britanniques de l'obligation de détenir un permis pour posséder quelqu'arme à feu (le port d'armes cachées sur soi nécessitant seul un permis), cette obligation ne continuant de s'appliquer, comme en 1919, qu'aux seuls «étrangers ». Cette célérité avec laquelle le gouvernement renoue avec sa politique traditionnelle en braquant son regard sur les «étrangers " vaut la peine d'être notée. D'ailleurs, en réponse aux remarques de certains députés soulignant le caractère circulaire de la législation, le ministre de la Justice ne se formalise pas pour affirmer que la loi de 1920 « avait un effet trop rigoureux, qu'elle prêt[ait] à des abus et caus[ait] aux citoyens [entendons les sujets britanniques] d'inutiles ennuis » ${ }^{53}$. Dans le dédale d'explications - certaines assez peu crédibles ${ }^{54}$ - mises en avant pour rendre compte de l'adoption de mesures aussi diamétralement opposées dans un si court laps de temps, figure également, aux dires de certains parlementaires, la question des pressions exercées par des manufacturiers d'armes à feu. Mais ce qu'il importe somme toute de retenir ici, c'est que le revirement de 1921 n'est qu'apparent, puisqu'il s'inscrit dans la continuité d'une politique logée à l'enseigne du double standard. Mais que dire alors des craintes précédemment formulées sur la dangerosité de tout homme armé, inconsidérément de son ethnicité ? En réalité, si en 1921 le législateur redonne aux sujets britanniques le droit de posséder à leur guise carabines, fusils de chasse et autres armes à feu, il prend toutefois la précaution d'insérer une clause (article 118, paragraphe 4, alinéa b) par laquelle le gouverneur en conseil est investi du pouvoir de réitérer en tout temps l'obligation universelle du permis de possession d'armes à feu. Ainsi, «en cas de nécessité résultant d'émeutes, explique le sénateur James Lougheed, la possession des armes à feu [pourra] être contrôlée $»^{55}$. Bref, d'une part, le réflexe xénophobe des autorités laisse son empreinte dans la loi, mais d'autre part, leur obsession que ne naisse quelque mouvement de déstabilisation sociale les amène à prévoir dans le Code des dispositions leur permettant de croire qu'ils pourront parer au pire.

Dans le dossier des armes à feu, l'amendement de 1921 sera en fait la dernière mesure législative adoptée par le gouvernement avant les années 1930. La question restera 
pourtant féconde en débats, mais elle n'arrivera pas à prendre corps dans une loi. À preuve, les interventions répétées du sénateur Belcourt d'Ottawa qui, à partir de 1921 et jusqu'en 1930, pressera sans relâche le gouvernement de pousser d'un cran la logique de la loi de 1920 qui, nous l'avons vu, rendait obligatoire pour tous les individus désireux de posséder une arme à feu de détenir d'abord un permis. Il parrainera ainsi au Sénat une cascade de projets de $l o i^{56}$ qui viseront à prohiber avec encore plus de rigidité la possession d'armes à feu et d'armes blanches dangereuses en en contrôlant de façon serrée l'importation, la fabrication et la vente ${ }^{57}$. Pour Belcourt, la possession d'une arme à feu devait être l'exception et jamais la norme. Or, l'opposition manifestée par les manufacturiers et les commerçants d'armes à feu, les craintes similaires de pertes financières exprimées par les compagnies ferroviaires qui transportent au Canada de nombreux touristes chasseurs et, enfin, la présence d'une interminable frontière canado-américaine qui rend illusoire le dessein de contrer l'entrée illégale d'armes au pays, seront des arguments invoqués ${ }^{58}$ pour laisser mourir au feuilleton parlementaire les propositions de réforme du sénateur Belcourt. La crise des années 1930, nous le verrons, contribuera néanmoins à faire renaître de ses cendres «l'esprit » de certaines de ses propositions.

Bref, en 1927, année où les statuts du Canada sont révisés, l'encadrement législatif qui prévaut en matière de contrôle des armes à feu correspond à celui qui avait été retenu dès 1919 et stigmatisait l'« étranger ». Malgré un louvoiement apparent, la politique gouvernementale adoptée reflète bien la sensibilité des décideurs à certaines réalités nouvelles : un paysage démographique changeant, caractérisé notamment par l'arrivée massive d'immigrants dont les sonorités linguistiques et les pratiques culturelles ne sont pas familières; l'émergence chez certains individus d'un patriotisme exacerbé, nourri par la guerre et enclin à exclure l'hétérogénéité; l'expression, enfin, sur la scène nationale de solidarités ouvrières que l'on craint de voir se modeler sur l'exemple communiste... Autant d'éléments qui convainquent le gouvernement qu'il serait dangereux de laisser les « étrangers » s'armer...

\section{3. - La transposition de la crise économique des années 1930 dans la législation du Code criminel afférente aux armes à feu}

42 Ce n'est pas un hasard si les années 1930, marquées par la pire dépression de l'histoire de l'économie canadienne, verront parallèlement une pluie d'amendements déferler sur le Code criminel pour réglementer la question des armes à feu. Nous avons déjà dit que 35 amendements ont été adoptés entre 1892 et 1939; or, 26 d'entre eux le furent entre les seules années 1933 et 1939, au fil de la promulgation de cinq lois : le 9 mai 1933 (chapitre 25), le 3 juillet 1934 (chapitre 47), le 23 juin 1936 (chapitre 29), le $1^{\text {er }}$

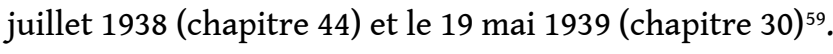

43 La détresse économique des années 1930 fut propice à l'éclosion du mécontentement, voire du désordre, social. La montée du chômage ( $26 \%$ de la main-d'œuvre en 1933) et la baisse abrupte des revenus (une chute de $48 \%$ entre 1928 et 1933) en contraignent alors plus d'un à recourir au "secours direct». Pour d'autres, c'est l'errance qui s'installe :

«En l'absence quasi totale de mesures de sécurité sociale - un maigre régime de pensions de vieillesse a vu le jour en 1927, mais rien n'est prévu pour aider les 
chômeurs, les malades et les démunis - les travailleurs ruraux et urbains dépendent de la charité d'organismes privés et publics. Beaucoup deviennent des vagabonds et se déplacent d'un bout à l'autre du pays en se hissant à bord de trains de marchandises, à la recherche de travail et de nourriture, ou pour tromper leur désœuvrement $»^{60}$.

L'ouverture de camps de travail en Colombie-Britannique sous le règne du gouvernement conservateur de Richard Bedford Bennett (1930-1935) s'inscrit d'ailleurs dans ce contexte. Dans ces camps qui sont de véritables ferments d'agitation sociale en raison même de l'impasse économique qu'ils symbolisent et sur lesquels se penchera d'ailleurs une commission d'enquête en $1935^{61}$, les travailleurs se rebellent sous l'influence syndicale de la Relief Camp Worker'sUnion, d'inspiration communiste... On connaît la suite: marche de 1800 travailleurs sur Ottawa, arrestation de leurs dirigeants à Régina, émeute, mort d'un policier... Faut-il souligner que le gouvernement a le parti communiste à l'œil, un parti fondé dans les années vingt et auquel la crise rallie alors des adhérents composés principalement de bas salariés et de certains groupes des milieux ethniques issus de contrées où le marxisme a déjà pris racine ? En réalité, les événements de Régina sont loin de constituer un cas isolé :

«Presque toutes les villes sont le théâtre de troubles, généralement moins graves que l'émeute de Régina. [...] Nerveuses, les autorités interviennent souvent trop vite, sous le prétexte qu'il est urgent d'enrayer la progression du communisme. L'arrestation, en 1931, des principaux dirigeants du parti communiste et la tentative d'assassinat par la suite, au pénitencier de Kingston, du chef du parti, Tim Buck [un immigrant britannique], incitent encore plus à penser que, pour toute réponse au mécontentement suscité par la crise, le gouvernement n'a rien trouvé mieux que « le talon de fer ", selon les termes mêmes de Bennett " ${ }^{62}$.

Les années trente sont donc des années troublées pendant lesquelles le gouvernement, désemparé, traque les communistes, réprime l'agitation ouvrière et endosse généralement la réaction allergique des milieux d'affaires à toute tentative nouvelle de syndicalisation ${ }^{63}$.

Cette lecture de la colère et du désespoir générés par la crise économique comme une menace communiste et une atteinte à l'ordre social en général, que fait le gouvernement, allait bien sûr être traduite dans le Code criminel. Dès le début des années 1930, la passation d'une première loi vient signer un remaniement complet de la grappe d'articles du Code touchant les armes offensives: la «Loi modifiant le Code criminel (Armes offensives)» sanctionnée le 9 mai 1933 (chapitre 25) abroge et remplace en effet les articles 118 à 129 du Code ${ }^{64}$. Cette loi vise d'abord et avant tout à exercer un contrôle plus sévère du port illégal d'armes à feu pouvant être dissimulées sur soi. Deux dispositions maitresses sont adoptées pour y parvenir. La première touche l'article 118 du Code - un article nouvellement configuré, puisqu'il ne traite désormais que des seules armes à feu - et prévoit l'assignation d'une peine considérablement aggravée : le port d'armes sans permis, considéré jusque-là comme une infraction passible d'une amende maximum de $100 \$$ ou/et une peine d'emprisonnement de trois mois, rend désormais son auteur coupable d'un acte criminel passible d'un maximum de 5 ans d'incarcération. "Demeure, boutique, entrepôt, bureau d'affaires ou terrain propres " échappent toujours (du moins pour les sujets britanniques) au champ de l'incrimination mais, et c'est là une nouveauté, le législateur, désireux d'empêchertout échappatoire, spécifie expressément l'obligation dudit permis pour quiconque «is carrying in any vehicle under his control, or of which he is an occupant, a pistol, revolver, or other firearm capable of being concealed upon the person $»^{65}$. La deuxième 
disposition insérée au Code pour circonscrire davantage la pratique du port illégal d'armes à feu porte sur l'article 119 qui, à l'alinéa (c), introduit l'obligation nouvelle pour le vendeur de détenir un permis de vente de pistolets et de revolvers ( formule $76 \mathrm{C} »)$. Le non-respect des clauses de l'article 119, lui aussi nouvellement configuré (il traite désormais du port d'armes offensives qui ne sont pas à feu, de possession d'armes à feu par les sujets étrangers et de vente d'armes), constitue une infraction qui peut valoir à son auteur une amende maximum de 100 \$ et/ou une incarcération d'une durée de trois mois.

Sévérité de la peine et introduction du permis de vente d'armes à feu sont donc, en 1933, les deux réponses principales faites par le législateur à des demandes de changements au Code criminel qui, pour la plupart, étaient toutefois beaucoup plus musclées et visaient, somme toute, un désarmement complet de la population, un désarmement qui serait passé, en l'absence de permis, par l'interdiction radicale de la possession, de l'importation, de la fabrication et de la vente de pistolets et de revolvers. La matérialisation intégrale de ces demandes aurait, comme en 1920, dépouillé les sujets britanniques de leur privilège de garder sans permis des armes à feu dans leurs maisons. Ces revendications, qui ne sont pas sans rappeler celles du sénateur Belcourt quelques années auparavant, proviennent principalement, cette fois, - et en grand nombre - des provinces de l'Ouest et elles sont le fait des chambres de commerce, des autorités municipales et des forces policières ${ }^{66}$. Ces groupes de pression qui sont, on le conçoit aisément, plus sensibles à l'agitation sociale, se disent inquiets de la montée de deux phénomènes qu'ils veulent contrer : le port illégal d'armes à feu et les attaques à main armée. Un sentiment d'insécurité que traduisent bien les propos suivants du député Reid :

«J'ai sous les yeux, s'exclame-t-il aux Communes, quelques statistiques relatives à Vancouver. Je crois que la Chambre s'étonnera d'apprendre que durant les onze premiers mois de 1932, il y a eu dans cette ville 411 attaques à main armée. La vie des gens n'est plus en sécurité là-bas. Quelques-uns de ces bandits sont si cruels qu'ils assomment au moyen de leurs revolvers les individus qu'ils ont attaqués et qui se trouvent à ne pas avoir d'argent. Ils quittent les villes dans des automobiles allant à grande vitesse et se rendent dans des endroits paisibles où, à la pointe du revolver, ils attaquent les marchands... ${ }^{67}$.

48 "The carrying of concealed weapons is becoming more and more general with the spread of banditry and robbery ", ajoutera à son tour le leader du Sénat.Que l'image «de bandits armés parcour[a]nt le pays» pour reprendre l'expression du député Hanbury soit fondée ou non est secondaire ${ }^{68}$; ce qui nous importe ici c'est le sentiment de menace qu'elle traduit chez ceux qui pendant ces années de misère, demandent ces amendements.

49 Si le gouvernement ne donne que partiellement suite aux changements souhaités en faisant valoir notamment que l'importation nécessite déjà un permis du ministère du Revenu national et que l'interdiction de la vente de ces armes dans les provinces pourrait soulever des problèmes d'ordre constitutionnel ${ }^{69}$, il a toutefois plus de difficultés à justifier la possession sans permis (pour les sujets britanniques) d'armes à feu dans les maisons. "Qu'est-ce qui empêcherait un individu d'une ville quelconque de garder continuellement chez lui une arme et de ne la sortir qu'au moment où il compte s'en servir?", proteste par exemple le député Stewart d'Edmonton qui ajoute qu'«il devrait exister des règlements très rigoureux, non seulement pour le port des armes, 
mais aussi pour leur possession $»^{70}$. Ce à quoi souscrivent entièrement les associations policières, comme en témoigne le commentaire suivant de l'un de leurs représentants :

«[...] at the present time, concealed weapons cannot be carried without a permit, nor can any firearms of any kind be possessed by an alien. In my opinion and in the opinion of many law enforcements officials, it would be very much better if firearms of any kind were not allowed to be possessed by any person whatever unless they had a permit $»^{71}$. « charbonnier est maître chez soi et l'Anglais aussi » et que « assurément, la défense de son foyer constitue le premier devoir de tout citoyen". Même s'il reconnaît la pertinence des arguments invoqués, il croit que sa stratégie d'intervention législative suffira à colmater les brèches :

« [...] d'après les rapports des associations de police des différentes villes du Canada, le nombre des personnes trouvées en possession de pistolets et de revolvers a considérablement augmenté depuis quelques années. C'est une chose courante aujourd'hui, lorsqu'un individu est arrêté pour un crime quelconque et qu'on le fouille au bureau de police de trouver sur lui un pistolet ou un revolver.[...]. On estime que si l'on restreignait la vente et si l'on prescrivait une peine plus sévère pour le port de ces armes, cela aurait pour effet de diminuer considérablement le trafic en question $»^{72}$.

51 Car il faut bien retenir ici que malgré les réticences du gouvernement à endosser l'intégralité des changements qui lui sont proposés, il n'hésite néanmoins aucunement à légiférer, ce qu'il n'avait pas fait depuis 1921 ! Il juge, lui aussi, la situation explosive, sauf qu'il persiste à s'enferrer dans sa logique du double standard, une logique suivant laquelle l'étranger reste toujours plus dangereux que les autres. Les Débats parlementaires de la période sont d'ailleurs émaillés de propos voulant resserrer le contrôle des étrangers. En fait foi, parmi d'autres, la remarque suivante du sénateur Charles E. Tanner :

«J'exprime [...] la conviction que plusieurs [des fauteurs de troubles de ce pays] sont des aubains qui n'ont pas du tout l'intention de devenir sujets britanniques. [...] Nous n'admirons pas tout ce qui se fait en Allemagne, mais je sais qu'un étranger qui entre dans ce pays est obligé de s'enregistrer immédiatement; [...] les autorités allemandes exercent un contrôle entier sur chacun de ceux qui entrent dans leur pays : on sait où il est, où il va, combien de temps il restera dans le pays, et pourquoi $»^{73}$.

Tanner clôt donc son allocution en proposant l'adoption du modèle allemand pour surveiller les «étrangers ». Rappelons ici que la prise triomphale du pouvoir par Hitler en Allemagne en 1933 ne provoqua pas de réaction dans le gouvernement canadien. Les propos du sénateur J. P. B. Casgrain qui, sur la question, prend ensuite la parole sont par surcroît teintés de démagogie :

«On prétend que plusieurs des aubains dans ce pays sont des agents soviétiques. $L a$ Revue des Deux Mondes a dit dernièrement [...] que, dans la seule ville de Toronto, il y a cent vingt-cinq hommes payés par l'Armtorg de New York et qui s'occupent uniquement de faire de la propagande pour les Soviets. [...] Ces agents auraient plus de difficultés à poursuivre leur propagande si tous les aubains étaient forcés d'avoir une carte d'identification.

[...] à Montréal [...] il y a eu dernièrement une série d'incendies criminels. Des églises ont été brûlées. [...] Je crois que le Sénat ferait bien d'encourager l'Administration actuelle à exiger l'identification de tous les aubains. Si un aubain n'a ni adresse, ni occupation connue, n'est-il pas sujet à déportation ? Quelques-uns 
de ces Bolchevistes reçoivent des secours de chômage et sont nourris aux frais $\mathrm{du}$ pays. L'effet en est très mauvais sur tout le reste de la population $»^{74}$. permis, à des armes est devenue pour ainsi dire sacrilège. La seule exception à laquelle souscriront les parlementaires sera de permettre « [...] à tout sportsman et chasseur de bonne foi d'entrer au Canada ou de traverser le territoire canadien, en ayant en sa possession un fusil de chasse, une carabine ou autre arme à feu de ce genre, ou des munitions pour cette arme, à la condition de traverser en continuation le territoire canadien, et d'en sortir, sinon de demander, sans retard déraisonnable, un permis [...] après entrée au Canada ${ }^{75}$. Cet amendement sera en fait adopté à l'instigation des compagnies ferroviaires qui s'occupent du transport de ces chasseurs sportifs et qui ne veulent pas voir leur clientèle se trouver en contravention entre le moment où est franchie la frontière canadienne et celui de l'arrivée au bureau des permis ${ }^{76}$.

de 1933, avons-nous dit, vise à encadrer plus sévèrement la question du port illégal d'armes à feu. Outre les clauses principales qui, pour y parvenir, remanient les articles 118 (peine) et 119 (vente) du Code, d'autres dispositions de la loi convergent dans le même sens. À l'instigation de la Chambre de commerce de Vancouver et donnant aussi suite à certains articles parus dans le journal The Vancouver Sun, le fait de porter cachée sur soi une arme à feu au moment de commettre un crime vaudra désormais à son auteur une peine de deux ans de prison, en sus de la sentence déjà prévue pour ledit crime dont il est reconnu coupable. L'aggravation de la peine, ici insérée à l'intérieur de l'article 122 du Code criminel, est réelle, puisque n'était prévue jusque-là qu'une amende de vingt à cinquante dollars ou une incarcération de trois mois $^{77}$. Dans le même esprit et à la demande des forces policières, il y aura dorénavant moins de personnes habilitées à délivrer des permis de port d'armes pour éviter qu'un individu qui s'est vu refuser l'octroi d'un tel permis puisse facilement du jour au lendemain refaire ailleurs une demande ${ }^{78}$. L'article 120 (1) du Code criminel prévoit ainsi que pourront désormais seuls remplir cette fonction « le commissaire de la Royale gendarmerie à cheval [sic] du Canada ou tout officier de cette gendarmerie dûment autorisé par le commissaire, ou tout individu autorisé par le procureur général d'une province $~^{79}$. Il fixe également les conditions qui pourront justifier l'obtention d'un permis : le demandeur devra convaincre l'officier responsable qu'il « [...] a besoin d'un pistolet ou revolver pour la protection de la vie ou de la propriété ou pour le tir à la cible dans un club de tir régulièrement organisé, approuvé par le gouverneur général de la province dans laquelle ce club est organisé $»^{80}$. Pour tout dire, de tous les changements apportés au Code criminel par la loi de 1933, un seul semble aller à contre-courant : il s'agit de l'abaissement de l'âge - de 16 à 12 ans - à partir duquel un mineur pourra légalement se voir aliéner une arme à feu ou à vent sans que celui qui a vendu ou donné l'arme ne soit coupable d'une infraction. Il s'explique en réalité de la façon suivante. Cet amendement a été suggéré à la Chambre des Communes par des députés qui firent valoir que cette interdiction faite aux enfants de moins de 16 ans d'utiliser de telles armes était systématiquement violée parce que la population en ignorait l'existence et que sa stricte application empêcherait, somme toute, un père de donner une arme à son fils pour aller chasser le petit gibier ${ }^{81}$. Des arguments qui n'ont donc rien à voir avec l'intention générale de la loi de 1933 qui est - par l'insertion notamment de peines plus sévères à des fins dissuasives - de réglementer le port des armes à feu pour faire baisser la criminalité et éviter que ne se lézarde un édifice social miné chaque jour davantage par la crise économique. 
55 La fébrilité législative de l'année 1933 ne semble pas avoir essoufflé le gouvernement canadien qui gardera son rythme de croisière l'année suivante. Dès le 3 juillet 1934, l'adoption d'une loi modifiant le Code criminel (chapitre 47) vient en effet resserrer encore davantage l'étau de la loi pénale. Quatre modifications principales sont alors apportées. La première et la plus importante rend désormais obligatoire l'enregistrement de tout pistolet ou revolver possédé par un individu. L'article 121A du Code criminel expressément créé à cet effet déclare ainsi :

«... coupable d'une infraction et passible, sur déclaration sommaire de culpabilité, d'une amende d'au plus cinquante dollars ou d'un emprisonnement pendant au plus trente jours, ou de l'amende et de l'emprisonnement à la fois, quiconque a en sa possession un pistolet ou revolver sans l'avoir enregistré en la manière ci-après prévue.

Le commissaire de la Royale gendarmerie à cheval [sic] du Canada ou tout officier dûment autorisé par lui, ou tout individu autorisé par le Procureur général de quelque province, doit enregistrer tous revolvers et pistolets faisant l'objet d'une demande d'enregistrement, et il doit aussitôt inscrire le nom, l'adresse et le métier ou profession de la personne qui fait la demande, le nom du propriétaire, l'usage, s'il en est, auquel le pistolet ou revolver est destiné, ainsi qu'une description complète de ce revolver ou pistolet ${ }^{82}$.

56 À partir de 1934, il ne suffit donc plus d'avoir un permis de port d'armes auquel s'ajoute, pour les étrangers, un permis distinct ${ }^{83}$ de possession d'armes à feu: tout possesseur de pistolet et de revolver doit dorénavant enregistrer ses armes. Le gouvernement souscrivit d'autant plus facilement à cet amendement demandé par la Gendarmerie royale ${ }^{84} q^{\prime}$ 'il lui permettait de forcer les sujets britanniques à enregistrer leurs armes, tout en continuant de les exempter de l'obligation de détenir un permis de possession d'armes. La deuxième modification apportée par la loi de 1934 touche à nouveau l'article $122 \mathrm{du}$ Code criminel amendé, nous l'avons vu, l'année précédente et elle prévoit que la sentence additionnelle de deux ans assignée à celui qui porte une arme pendant la perpétration d'un acte criminel pour lequel il est reconnu coupable, sera désormais considérée comme une peine "minimum». Troisième changement apporté en 1934 : sera désormais coupable d'infraction celui qui, sans permis («formule $76 \mathrm{D} »)$, achète des pistolets ou revolvers pour les revendre ou, s'il en est muni, omet de tenir un registre descriptif de ses achats. La Chambre des Communes s'était d'ailleurs faite l'écho de certaines plaintes des forces policières sur la question : «... je me suis souvent demandé, dira ainsi un député, pourquoi l'on permettait aux marchands d'occasion de remplir leurs vitrines de revolvers usagés qui se vendent d'un dollar et demi à deux dollars $»^{85}$. Enfin, dernier amendement, celui qui répare une arme est dorénavant tenu - comme le vendeur - d'inscrire tous les renseignements afférents à cette réparation (description de l'arme, nom du propriétaire, date de la réparation, etc.). Dans les deux derniers cas, les dispositions de l'article 119 (1) (e) - «omission d'enregistrer la vente ou la réparation » - et (h) - « achat pour revente » - dont il est ici question, prévoient une peine maximum de $100 \$$ et/ou trois mois de prison.

La troisième loi modifiant le Code criminel et recoupant la question des armes à feu pendant les années trente est promulguée le 23 juin 1936 (chapitre 29). L'idée qui soustend ces nouveaux changements est toujours la même: essayer d'empêcher les individus de porter illégalement, cachées sur eux, des armes à feu en renforçant l'attirail pénal du Code tout en agrandissant toujours davantage le champ d'incrimination pour contrer toute échappatoire. S'inscrit dans cette démarche l'amendement apporté à l'article 118 (ajout de l'alinéa c) du Codecriminel qui précise de 
façon spécifique que la peine prévue de cinq ans d'emprisonnement pour port d'armes à feu sans permis s'appliquera également au "fusil de chasse à canon raccourci ou [à] une carabine dont le canon a moins de vingt pouces de longueur ». Quelques années plus tôt, la question des fusils de chasse tronqués avait été soulevée à la Chambre des Communes: plus puissantes et plus dangereuses que les revolvers, avait-on dit, ces armes sont portées de façon courante dans l'Ouest, ce dont s'inquiètent les cercles policiers. Soucieux de les englober dans la formulation de l'article 118 en 1933, le législateur avait alors inséré après les mots "pistolet " et "revolver» le segment suivant : « ou autre arme à feu pouvant être dissimulée sur soi $»^{86}$. Or les mailles du filet devaient être perçues comme étant encore trop lâches puisqu'en 1936, à la suite de recommandations en ce sens des forces policières, le gouvernement légifère cette fois de façon expresse sur le sujet ${ }^{87}$.

Autre amendement apporté en 1936: l'article 120 (1) du Code criminel prévoit désormais un cas additionnel justifiant l'octroi d'un permis de port de pistolet ou de revolver, lorsque ladite arme sert d'outil de travail au demandeur ${ }^{88}$. Les trappeurs, les chirurgiens vétérinaires, etc., sont ici visés ${ }^{89}$. Le travail est donc ajouté aux critères d'attribution des permis, à côté de la protection de la vie privée ou de la propriété et de la pratique du tir à la cible dans un club homologué.

59 S'il y a accalmie législative sur la question des armes à feu en 1937, l'agitation parlementaire reprend de plus belle l'année suivante. La loi modifiant le Code criminel sanctionnée le $1^{\mathrm{er}}$ juillet 1938 (chapitre 44) est en effet chargée : étrangers, mineurs, permis, enregistrement, autant de thèmes différents auxquels elle apporte des correctifs. À la demande du Bureau du procureur général de la Saskatchewan, demande fortement appuyée par la gendarmerie royale, le gouvernement décide qu'il incombera désormais à l'individu non naturalisé qui est accusé de possession d'arme à feu de faire «la preuve qu'il n'est pas un sujet étranger». La difficulté qu'aurait éprouvée la Couronne à faire cette preuve serait à l'origine de cette nouvelle discrimination envers les étrangers ${ }^{90}$. Autres réformes apportées en 1938, suite aux pressions exercées en ce sens par les intervenants policiers: d'abord, l'introduction du "permis d'achat d'un pistolet, revolver ou autre arme offensive » («formule 76E ») avec obligation faite au vendeur, à l'alinéa (d) de l'article 119 (1), d'en prendre acte ${ }^{91}$; ensuite, l'obligation, nouvellement inscrite à l'article $121 \mathrm{~A}$ (3), de procéder à un enregistrement quinquennal de tous les revolvers et pistolets, une mesure qui se veut complémentaire à l'enregistrement déjà prévu audit article depuis $1934^{92}$. Dans la logique de l'adoption de ces mesures d'enregistrement destinées à dresser des fiches signalétiques fidèles, la Gendarmerie royale du Canada secondée par le corps de police de Toronto, obtient du législateur de rendre punissable quiconque "altère, détériore ou fait disparaitre le numéro de série d'un fabricant sur quelque pistolet, revolver ou autre arme à feu susceptible d'être cachée sur la personne ${ }^{93}$. Là ne s'arrête pas, en 1938, le travail de remodelage du Code criminel au chapitre des armes à feu. Deux autres amendements doivent encore être signalés. Le premier touche l'article 122 et spécifie que l'application de la peine additionnelle de deux années minimum, infligée pour port d'armes à feu lors de la perpétration d'un acte criminel, s'étend désormais explicitement au port de la carabine et du fusil de chasse ${ }^{94}$. Le second modifie l'article 126 et vise les mineurs. On se souvient qu'en 1933, l'âge à partir duquel un mineur pouvait se voir aliéner une arme avait été abaissé de 16 à 12 ans, un amendement dont l'esprit, nous l'avons dit, était en porte-à-faux au sein d'une loi empreinte de sévérité. Or, selon de nombreux groupes de pression ${ }^{95}$, ces assouplissements de 1933 se sont traduits par un nombre élevé 
d'accidents reliés surtout, doit-on préciser, à l'usage incontrôlé des armes à vent. Bref, interdiction sera faite à partir de 1938 d'aliéner des armes à feu ou à air comprimé «à un mineur âgé de moins de 14 ans qui n'est pas détenteur d'un permis » ce qui est un élément nouveau ${ }^{96}$. Les mineurs avaient jusque-là échappé au travail de révision serrée poursuivi par le pouvoir législatif depuis le début de la décennie. Ils ne font dorénavant plus exception à la règle.

60 La cascade d'amendements afférents aux armes à feu qui ont ponctué les années trente n'est donc nullement fortuite. La gangrène économique qui ronge alors le pays tend à déstabiliser le tissu social. La misère s'accompagne, en effet, de désespoir, de colère et de mobilisation sociales, et pour certains, du recours au crime. Dans ce climat d'insécurité générale où les drames individuels côtoient le désarroi collectif, l'élite politique est inquiète... Elle est d'autant plus nerveuse qu'elle est régulièrement alertée par les forces policières - le groupe de pression le plus constant - de même que par les gens d'affaires et les autorités municipales, qui lui demandent de consolider l'ordre social en adoptant dans le Code criminel des mesures plus musclées pour faire échec au crime. Ces appels répétés ne restent pas sans écho, comme en témoigne la pluie d'amendements relatifs aux armes à feu, qui figent dans la loi les craintes des décideurs. Le gouvernement passe donc à l'attaque et, un peu comme s'il investissait un lieu, circonscrit de toutes parts la pratique du port illégal d'armes à feu, brandit l'arme de la dissuasion pénale, somme les possesseurs de pistolets et revolvers d'enregistrer leurs armes (1934) et fait intervenir dans le feu de l'action toute une batterie de nouveaux permis. Aux deux permis de port d'armes offensives (à feu et autre) pouvant être cachées sur soi, s'ajoutent en effet : un permis distinct de possession d'armes à feu pour les étrangers (1933), un permis de vente de pistolets et revolvers (1933), un permis d'achat pour revente de pistolets et revolvers (1934), un permis d'achat d'un pistolet, revolver ou autre arme offensive (1938) et, enfin, un permis de possession et de port d'arme à feu ou de fusil à vent par un mineur âgé de moins de 14 ans (1938).

61 Telle est donc la structure du contrôle des armes à feu que l'on trouve à l'aube de la Deuxième Guerre mondiale. Or, quitte à déborder quelque peu le cadre chronologique de notre étude, soulignons rapidement que cette structure traversera non seulement à peu près intacte les années quarante, mais demeurera encore bien visible après la révision complète des articles du Code concernant les armes offensives qui sera faite en 1951. Signalons, néanmoins, trois changements notables. Le premier sera l'élimination, en 1950, de la vieille clause teintée de xénophobie interdisant aux seuls étrangers la possession de quelqu'arme à feu sans avoir préalablement obtenu un permis. Faut-il voir dans cette disparition une quelconque révolution des mentalités législatives? Non. C'est que la toile du Code criminel est alors tissée avec une telle soliditél'enregistrement obligatoire des pistolets et revolvers pour tous les propriétaires depuis 1934 annonçait d'ailleurs déjà l'inutilité de cette disposition ségrégative - que, non seulement, cette clause ne renforce plus en rien la sécurité nationale, mais qu'elle se traduit encore par une augmentation stérile du travail des forces policières. D'où la demande de la Gendarmerie royale de l'abroger ${ }^{97}$. La deuxième modification à retenir sera l'introduction, en 1951, d'une nouvelle disposition autorisant un agent de la paix à faire une perquisition "sans mandat » dans « un véhicule ou [...] dans des lieux autres qu'une maison d'habitation $»^{98}$. Enfin, le dernier changement d'importance en 1951 sera l'établissement d'un fichier central («central registry») sous la supervision du commissaire de la Gendarmerie royale du Canada mentionnant chaque certificat d'enregistrement d'arme à feu ${ }^{99}$. Cette révision de 1951 qui simplifiera, en effet, toute la 
procédure en matière d'armes (enregistrement, port, possession, vente), viendra donc surtout, pour reprendre Dandurand, "réorganiser le texte des articles du Code concernant les armes offensives $»^{100}$. En d'autres termes, l'essentiel de l'échafaudage législatif en matière de contrôle des armes à feu était déjà bien monté à la fin des années trente.

\section{Conclusion}

Dans le dossier du contrôle des armes à feu, le premier demi-siècle d'existence du Codecriminel canadien témoigne donc d'une grande sensibilité à l'évolution conjoncturelle d'un jeune pays en train de se former et de se complexifier. L'importance de l'immigration à partir du tournant du siècle a été déterminante dans l'ajout des premiers amendements apportés au Code: aboutée, dans l'esprit des décideurs, à la hausse de la criminalité, elle s'est alors traduite, pour l'essentiel, par une réglementation plus stricte et plus sévèrement punie du port illégal d'armes offensives et par l'interdiction d'aliéner une arme à un individu non détenteur d'un permis de port d'armes. D'autres phénomènes contemporains laissèrent ensuite leur empreinte dans le Code. L'éclatement de la Première Guerre mondiale et l'émoi semé par les clameurs ouvrières sur les scènes nationale et internationale amènent en effet le gouvernement à stigmatiser législativement les «étrangers ». Ils deviennent les seuls à ne pouvoir posséder - même dans leur propre demeure - quelqu'arme sans permis.

Ce premier resserrement du contrôle des armes à feu allait être accentué dans la tourmente économique des années trente. Au rang des interventions gouvernementales visant à empêcher l'explosion de la soupape de sûreté d'un ordre social jugé en péril se trouve, en effet, l'adoption d'une législation criminelle aux dents plus acérées qui passe, entre autres, par une aggravation de la peine pour port illégal d'armes à feu, par l'introduction d'une série de nouveaux permis et par l'enregistrement obligatoire de tout pistolet ou revolver possédé par un individu. Lorsqu'éclate la Seconde Guerre mondiale, le Canada est donc doté d'une structure de contrôle des armes à feu bien ancrée dans le paysage juridique canadien.

\section{BIBLIOGRAPHIE}

Avery, D., « Dangerous Foreigners ». European Immigrant Workers and Labour Radicalism in Canada 1896-1932, Toronto, McClelland \& Stewart Limited, 1979.

Brown, C. (Dir.), Histoire générale du Canada, Édition française dirigée par Paul-André Linteau, Montréal, Éditions du Boréal, 1990.

Brown, R. C., Cook, R., Canada 1896-1921 : A Nation Transformed, Toronto, McClelland \& Stewart, 1974.

Cellard, A., Pelletier, G., La construction de l'ordre pénal au Canada, 1892-1927 : approches méthodologiques et acteurs sociaux, Déviance \& Société, 1999, 23, 4, pp. 367-393. 
Dandurand, Y., Description de l'évolution de la partie positive du Code criminel canadien, 1892-1955, document de travail, ministère de la Justice du Canada, 1982.

Foran, M., Calgary: An Illustrated History, Photographs assembled by Edward Cavell, Toronto, The History of Canadian Cities Series, James Lorimer \& Company, Publishers and National Museum of Man, National Museums of Canada, Toronto, 1978.

Friedland, M. L., A Century of Criminal Justice. Perspectives on the Development of Canadian Law, Toronto, Carswell Legal Publications, 1984.

Hamelin, J., Montminy, J.-P., Québec, 1896-1929 : une deuxième phase d'industrialisation, in Dumont, F., Hamelin, J., Harvey F., Montminy, J.-P. (Dir.), Idéologies au Canada français 1900-1929, Québec, Collection « Histoire et sociologie de la culture », Les Presses de l'Université Laval, 1974, pp. 15-28.

Harvey, F., Révolution industrielle et travailleurs, Montréal, Boréal Express, 1978.

Henderson, G. F., Federal Royal Commissions in Canada 1867-1966, A Checklist, Toronto, University of Toronto Press, 1967.

Kealy, G. S., 1919 : The Canadian Labour Revolt, in Palmer, B. D., (Ed.), The Character of Class Struggle: Essays in Canadian Working-Class History, 1850-1985, Toronto, McClelland \& Stewart, 1986, pp. 90-114.

Momryk, M., A century of Ukrainian life in Canada, The Archivist, July-September 1991, 18, 2, pp. 14-15.

Page, R., La guerre des Boers et l'impérialisme canadien, Ottawa, La Société historique du Canada, Brochure historique $\mathrm{n}^{\circ} 44,1987$.

Strong-Boag, V., « Setting the stage » : National organization and the Women's Movement in the Late 19th Century, in Trofimenkoff, S. M., Prentice, A., (Eds.), The Neglected Majority : Essays in Canadian Women's History, Toronto, McClelland \& Stewart, 1977, pp. 87-103.

\section{NOTES}

2. L'on trouvera davantage de précisions sur cette question dans Cellard, Pelletier (1999, p. 369).

3. Cellard, Pelletier (1999, pp. 371-372).

4. Le 6 décembre 1989, un tireur fou armé d'un fusil semi-automatique tuait sauvagement quatorze jeunes femmes à l'École polytechnique de Montréal. Le "meurtrier antiféministe ", pour reprendre une expression utilisée alors dans la presse écrite, blessa également plusieurs autres personnes avant de s'ôter la vie.

5. Momryk (1991, p. 14).

6. Brown (1990, pp. 458-459).

7. Tout ce qui précède s'inspire de Brown (1990, pp. 453-458 et 510-516). Voir aussi Hamelin, Montminy (1974). Soulignons ici qu'en plus des produits agricoles, les matières premières canadiennes sont elles aussi alors davantage recherchées par les pays industrialisés.

8. Page (1987).

9. Brown (1990, pp. 499-500).

10. Foran (1978, p. 176).

11. Les données sur la croissance urbaine, mises à part celles sur Calgary, sont tirées de Brown (1990, p. 470).

12. Les travailleurs ne sont pas les seuls au cours de ces années à consolider leurs forces au sein d'organisations pan-canadiennes. En témoignent la nouvelle stature nationale de certains 
regroupements féminins et de certaines associations créées au sein du monde des affaires de même que la conclusion d'alliances plus grandes entre les Églises protestantes. Voir Strong-Boag (1977).

13. Code criminel canadien [dorénavant CC], 1892, chapitre 29, article 3 (f) : « L'expression arme offensive comprend tout fusil ou autre arme à feu ou fusil à vent, ou toute partie de ces armes, et toute épée, lame d'épée, bayonnette, pique, pointe de pique, lance, pointe de lance, dague, poignard, couteau ou autre instrument propre à trancher ou percer, et toutes jointures de métal, ou autres armes meurtrières ou dangereuses, et tout instrument ou chose destinée à servir d'arme, et toutes munitions qui peuvent être employées avec une arme quelconque ».

14. CC, 1892, chapitre 29, article 105 («Porter un pistolet sans cause raisonnable »), article 112 («Exception quant aux soldats, etc. ») et article 107 (« Porter une arme lors d'une arrestation »).

15. Harvey (1978, p. 150). Quant aux femmes exerçant les mêmes métiers, elles gagnaient respectivement 4 \$ et $3 \$$ en moyenne par semaine.

16. CC, 1892 , chapitre 29 , articles 110 et 111 . L'amende maximum pour port de couteaux à gaine est de $40 \$$.

17. L'article 106 (1) couvre la question des mineurs et l'article 106 (2) celle du registre de transactions.

18. CC, 492-493 : «S. R. C. [Statuts révisés du Canada], Chapitre 148, Acte concernant l'usage abusif des armes à feu et autres » et «S. R. C., Chapitre 149, Acte concernant la saisie des armes gardées dans un but dangereux ".

19. En 1906, la numérotation des articles du Code criminel canadien a complètement changé. Les anciens articles 110 et 111 de 1892 sont ainsi devenus les articles 123 et 124 .

20. Débats de la Chambre des Communes du Canada [dorénavant DCC], 21 avril 1909, pp. 4879-4880; Débats du Sénat du Canada [dorénavant DS], 18 mai 1909, pp. 729-730.

21. Le solliciteur général du Canada, dont le poste fut créé en 1888, était alors un adjoint du ministre de la Justice. Précisons qu'il est à la tête d'un ministère distinct depuis 1966. Il est responsable des prisons et des pénitenciers, des maisons de correction, des libérations conditionnelles et des remises de peine, ainsi que de la gendarmerie royale du Canada.

22. DCC, 12 mai 1909, p. 6706.

23. L'article 105 de 1892 est devenu l'article 118 à partir de 1906.

24. L'ancien certificat d'exemption ne valait que pour les armes à feu et les armes à air comprimé et non pour les armes blanches dont le port, nous l'avons vu, était prohibé par l'article 110 de 1892 devenu l'article 123 en 1906 et qui fut amendé en 1909. Notons que les dispositions du Code relatives aux armes blanches se retrouveront davantage à l'intérieur de l'article 118 à partir de 1913 et de l'article 119 à partir de 1933.

25. Cette infraction était auparavant couverte par l'article 106 (2) de 1892 devenu l'article 119 (2) en 1906 et la peine prévue était une amende de $25 \$$. Il y a en fait ici transfert de contenu d'un article à un autre.

26. La question des permis ne s'applique tout simplement pas aux mineurs de moins de seize ans puisque l'interdiction de leur aliéner des armes à feu est totale.

27. DCC, 16 May 1913 (version anglaise), p. 10071 (nous donnons exceptionnellement ici la version anglaise des Débats parce qu'il y a un contresens dans la traduction française).

28. Cet amendement édicté le 7 juillet 1919, chapitre 46, article 2, porte sur l'article 118 (d1) du Code criminel.

29. Archives nationales du Canada [dorénavant ANC], RG 13, vol. 2160, dossier 776/1919, lettre et résolution du Conseil municipal de Guelph au Premier ministre Robert Borden, 5 février 1919.

30. DCC, $1^{\text {er }}$ juillet 1919, p. 4513.

31. ANC, RG 13, vol. 2159, dossier 351/1919, memorandum de l'Acting chief commissioner of police du Canada à Mr Varcoe, Justice Department, Ottawa, 15 mai 1919. 
32. En plus du Premier ministre Borden, l'équipe ministérielle unioniste (12 octobre 1917-10 juillet 1920) compte quinze Conservateurs, neuf Libéraux et un Travailliste.

33. DCC, $1^{\text {er }}$ juillet 1919, p. 4514 : «Les renseignements que j'ai en main, dit Meighen, suffiraient à convaincre les plus incrédules qu'il est grandement temps de veiller à ce qu'il n'y ait plus d'armes cachées, non par des dizaines ou même par des centaines, mais par des milliers de personnes en ce pays, la plupart des étrangers, et par un petit nombre de sujets britanniques ».

34. DCC, $1^{\text {er }}$ juillet 1919, pp. 4512-4513.

35. Avery (1979, p. 66) : «The outbreak of war in August 1914 forced the Dominion government to adopt a comprehensive set of guidelines for dealing with the enemy alien resident of the country. Of the persons classified as enemy aliens there were 393,320 of German origin, 129,103 from the Austro-Hungarian Empire, and several thousands from Bulgaria. [...] By end of the war over 80,000 enemy aliens had been registered, [...] 8,579 of these were actually interned. [...] These 8,579 prisoners of war were located in some twenty-four different camps...[...] Although there were very few incidents of sabotage or espionage on the home front during the war, enemy aliens soon became the object of intense Anglo-Canadian hostility ».

36. L'article 75A du Code criminel fut créé le 15 avril 1915, chapitre 12, article 2 - Certains sénateurs s'opposèrent en vain à l'adoption de telles propositions : « C'est parler contrairement à tous les principes de la loi anglaise que de dire qu'une personne accusée d'un crime doit prouver son innocence », protestera entre autres le sénateur Choquette (DS, 31 mars 1915, p. 305).

37. «Associations, Organizations, Societies or Groups Declared to Be Unlawful Associations » : ANC, RG 13, vol. 2809, dossier 136601 : PC 2384.

38. Brown, Cook (1974, p. 313).

39. Une équation que, de surcroît, l'encourageront à faire les déclarations de certains leaders syndicaux particulièrement fougueux et habiles à souffler sur la braise de la menace communiste : «I am a Bolshevist, dira ainsi l'un d'eux, and I will warn these two governments that trouble is coming and the men will have what belong to them »; cité in Kealy (1986, p. 92).

40. Cité in Dandurand (1982, p. 276).

41. Avery (1979, p. 75). En plus de l'Ordre en Conseil de 1918 déclarant illégales une liste d'organisations «ethniques ", un autre vint museler la presse de langue étrangère : "... the foreign-language press was suppressed...[...] The newspapers most affected by the censorship regulations were those which published in the languages categorized as "enemy alien »: German, Bulgarian, Ukrainian, Estonian, Ruthenian, Hungarian, Turkish, Russian, Finnish, Croatian, and Livonian ».

42. DCC, $1^{\text {er }}$ juillet 1919, pp. 4513, 4515.

43. DCC, $1^{\text {er }}$ juillet 1919, pp. 4514, 4515.

44. DCC, $1^{\mathrm{er}}$ juillet 1919, p. 4514.

45. Le changement relatif au transport d'armes cachées dans un sac porte sur l'article 118 (1) (a) du Code criminel. La question de la juridiction dans laquelle est valable un permis réfère à 118 (2) et celle des agents d'immigration pouvant porter des armes sans permis à 118 (5).

46. ANC, RG 13, vol. 2159, dossier 351/1919, lettre du surintendant de l'Immigration au ministre de l'Immigration et de la Colonisation, James Alexander Calder, 30 avril 1919.

47. ANC, RG 13, vol. 2171, dossier 2117/1921, lettre du Major général Fiset, sous-ministre de la Milice et de la Défense, à Francis H. Gisborne, «Parliamentary Counsel », Chambre des Communes, Ottawa, 12 décembre 1918.

48. Amendement du 10 novembre 1919, chapitre 12, article 1 . Référence est faite ici à l'article 118 (d1) du Code criminel.

49. L'article 118 du Code criminel ainsi amendé déclare donc dorénavant coupable d'infraction quiconque «(a) possède un canon, une mitrailleuse, une carabine, un fusil, un revolver, un pistolet, une bombe ou une autre arme à feu ou quelque fusil à vent ou invention ou dispositif pour assourdir ou arrêter le bruit de la détonation d'une arme à feu, sans avoir de permis à cet 
effet, ce permis pouvant être émis de la même manière par les mêmes personnes, et autant que possible suivant la même formule que pour les autres permis dont il est fait mention au présent article. Toutefois, nul sujet britannique n'est requis d'obtenir un permis relativement à un fusil de chasse qu'il a actuellement en sa possession ».

50. DCC, 10 juin 1920, pp. 3480, 3481.

51. DCC, 10 juin 1920, p. 3482.

52. DS, 1920, p. 733-734. Cité in Dandurand (1982, p. 216).

53. DCC, 6 mai 1921, p. 3057.

54. Un député prétendra ainsi que : «L'an dernier, la Chambre a adopté un amendement au Code criminel qui n'a pas été bien compris par les membres de la Chambre qui étaient présents ", DCC, 26 mai 1921, p. 3967.

55. DS, $1^{\text {er }}$ juin 1921, pp. 754-755.

56. Ce sont les projets de loi M3 (1921), Q3 (1926), A (1927), B (1928), B (1929). En 1930, les amendements défendus par Belcourt sont intégrés à l'intérieur du projet de loi $\mathrm{n}^{\circ} 138 \mathrm{du}$ ministre de la Justice pour en être ensuite écartés. Cette croisade législative du sénateur Belcourt est fort bien documentée in Dandurand (1982, pp. 220-226).

57. Dans son projet de loi M3 de 1921 antérieur, précisons-le, à l'adoption de la loi du 4 juin 1921 qui viendra au contraire assouplir la loi en faveur des sujets britanniques, Belcourt s'appuie sur la parution de nombreux articles éditoriaux (DS, 3 mai 1921, pp. 418-425) pour prôner d'abord l'interdiction quasi absolue de l'importation, fabrication et vente de ces armes. À partir de 1926, il suggère d'accrocher le tout à un système sévère de permis accompagné de lourdes peines.

58. Ils le sont notamment par le sénateur James Lougheed, leader du gouvernement. Voir : DS, 30 mai 1921, p. 686.

59. En réalité, la concentration législative est encore plus forte compte tenu du fait que la loi de 1939 ne contient qu'un seul amendement touchant les armes à feu (l'article 125 du Code criminel) et que l'objet de cet amendement est simplement « to correct a clerical error » (Projet de loi $n^{\circ}$ 90, article 4, First Reading, April 11, 1939, Explanatory Notes).

60. Brown (1990, p. 538). On trouvera les chiffres sur le chômage et la baisse des revenus à la page 535.

61. "Commission to investigate and report on all any complaints in reference to the management of the relief camps for single homeless men in the province of British Columbia ». Voir Henderson (1967, p. 128).

62. Brown (1990, pp. 538-539).

63. La majorité des renseignements qui précèdent sont tirés de Brown (1990, p. 533 et suiv.).

64. Certes, comme l'explique lui-même le ministre de la Justice Guthrie, ces articles ne sont pas tous remplacés par de nouvelles dispositions. Ainsi, le contenu de certains articles ou de parties d'articles peut tout simplement se retrouver sous une nouvelle numérotation : c'est le cas, par exemple, de l'article 121 de 1927 («Porter pistolet ou fusil à vent avec l'intention de blesser quelqu'un ») dont le contenu se retrouve en 1933 sous l'article 123 avec comme seul changement l'utilisation de l'expression "arme offensive » en remplacement de "pistolet ou fusil à vent". Mais dans l'ensemble, il n'en demeure pas moins qu'il s'agit ici d'un remaniement substantiel.

65. Cette criminalisation expresse du transport de telles armes à l'intérieur de véhicules visait à empêcher le coupable d'alléguer qu'il ne portait pas d'arme sur sa personne. Cette idée est exprimée dans : DCC, 29 mars 1933, p. 3496 (propos de l'hon. M. Elliot) et dans : ANC, RG 13, vol. 2223, dossier 1064/1933, lettre de R. B. Graham au sous-ministre de la Justice, W. Stuart Edwards, 13 janvier 1932.

66. ANC, RG 13, vol. 2223, dossier 1064/1933. Ce dossier regorge de demandes formulées par ces groupes de pression. Voir notamment: lettre de l'éditeur du Vancouver Sun au ministre du Commerce, Henry Herbert Stevens, 10 mars 1932; lettres de C. C. Delbridge, Police Commissioner of the City of Vancouver, au député Robert Rogers, 7 avril et 17 octobre 1932; lettres du 
Vancouver Board of Trade au ministre de la Justice, 19 mars 1932 et 23 janvier 1933; lettre du Board of Police Commissioners de Vancouver au ministre de la Justice, 18 octobre 1932. Voir encore DCC, 29 mars 1933, p. 3488ss.

67. DCC, 29 mars 1933, pp. 3491-3492.

68. D'autant plus que les données utilisées pour soutenir cette image sont pour le moins insuffisantes et précaires.

69. DCC, 29 mars 1933, p. 3489.

70. DCC, 29 mars 1933, p. 3497.

71. ANC, RG 13, vol. 2223, dossier 1064/1933, lettre de H. P. Reed, Police Magistrate (Russell, Manitoba), au ministre de la Justice Guthrie, 20 juillet 1932. En réalité, l'amendement de 1933 stipule que les étrangers ne peuvent posséder sans permis fusil de chasse, carabine «ou autre arme à feu de ce genre ", mais nulle part n'est-il fait mention expresse - comme c'était le cas auparavant - $d u$ revolver et $d u$ pistolet. Des plaintes fusèrent pour que l'interdiction de possession soit étendue aux pistolets et revolvers (ANC, RG 13, vol. 2223, dossier 1064/1933 : lettre de E. Bayly, Deputy Attorney General de Toronto, au sous-ministre fédéral de la Justice W. S. Edwards, 10 juillet 1933; lettre du Deputy Attorney-General de Winnipeg au sous-ministre fédéral de la Justice, W. S. Edwards, $1^{\mathrm{er}}$ août 1933; lettre de J. Russell, Serjeant, B. C. Police, au sénateur A. E. Planta, Nanaimo, B.C., 11 décembre 1933; lettre de Oscar Orr, City Prosecutor, Vancouver, au sous-ministre fédéral de la Justice, 27 décembre 1933; lettre de J. H. MacBrien, Commissioner RCMP, au ministre de la Justice Hugh Guthrie, 19 janvier 1934). Le 5 août 1933, dans une lettre qu'il adressait au procureur général adjoint de Winnipeg, G. P. Plaxton (ministère fédéral de la Justice), tentait d'expliquer ainsi la raison de cette omission : «It is quite true that an alien is not required to have a permit for a revolver if the revolver remains in his house. [...] it was considered undesirable to have a permit being issued [...] to enable an alien to have a revolver in his house when he would almost certainly be refused a permit to carry a revolver [...] It may be that we were wrong ». Le 3 juillet 1934 (chapitre 47, article 1), «l'omission » de 1933 était rectifiée: "l'étranger » ne pouvait posséder aucune arme à feu sans permis, mention spécifique étant dorénavant faite du pistolet et du revolver (amendement à l'article 119 (1) (b) du Code criminel).

72. DCC, 29 mars 1933, p. 3389.

73. DS, 6 avril 1933, p. 404.

74. DS, 6 avril 1933, p. 405.

75. Amendement du 9 mai 1933, chapitre 25, article 1. Référence est faite ici à l'article 119 (1) (b) du Code criminel.

76. DS, 10 avril 1933, p. 415 (propos du sénateur Lynch-Staunton) et 11 avril 1933, p. 424 (propos du sénateur Meighen).

77. Le contenu de l'article 122 du Code criminel amendé en 1933 renvoie à celui de l'ancien article 120 de 1927. Pour ce qui est des instigateurs, voir ANC, RG 13, vol. 2223, dossier 1064/1933, lettre du Vancouver Board of Trade au ministre de la Justice, 19 mars 1932 et extraits du The Vancouver Sun, 10 mars 1932.

78. ANC, RG 13, vol. 2223, dossier 1064/1933, lettre du Chief Constable Martin Bruton à A. Blackwood, Deputy-Attorney General de la Saskatchewan, 23 février 1933 : «Under the present law, écrit-il, if I refuse an applicant a permit to purchase a weapon, he is at liberty to go to the office of the Royal Canadian Mounted Police, the Police Magistrate or the Sheriff, and make application for the required permit without stating the same has already been refused by the Chief Constable ». Dès 1926, nous trouvions une requête en ce sens du Secretary-Treasurer de la Chief Constables' Association of Canada au ministre de la Justice Ernest Lapointe (ANC, RG 13, vol. 2194, dossier 443/1928).

79. La question des personnes pouvant émettre des permis était, avant l'amendement de 1933, couverte par l'article 118 (2) du Code criminel. 
80. Les pressions d'organismes comme le Toronto Revolver Club dont les membres se recrutent parmi les employés des banques et autres institutions similaires, sont responsables de l'insertion de la référence aux clubs de tir à la cible. Voir ANC, vol. 2223, dossier 1064/1933, lettre de Chester B. Hamilton, President du Toronto Revolver Club, au sénateur Arthur Meighen, 7 avril 1933. Et Meighen suggèrera d'ailleurs lui-même ledit amendement aux membres du Sénat: DS, 10 avril 1933, p. 418 (référence au projet de loi no 53 de la Chambre des Communes).

81. La question des armes et des mineurs est couverte en 1933 par l'article 125 du Code criminel. Voir DCC, 29 mars 1933, pp. 3502-3507.

82. Loi du 3 juillet 1934 , chapitre 47 , article 4.

83. Nous écrivons « distinct " parce qu'avant 1933, le même permis (suivant la «formule 76 ») était utilisé pour port d'armes offensives et pour possession d'armes à feu par les étrangers. En 1933, on retrouve trois permis différents se rapportant au port et à la possession d'armes à feu : un permis de port de pistolet ou revolver [ou autre arme à feu pouvant être dissimulée sur soi] (suivant la «formule $76 »$ ); un permis de port d'arme autre qu'un pistolet ou revolver ou autre arme à feu (suivant la «formule $76 \mathrm{~A} »)$; un permis de possession d'armes à feu pour les étrangers (suivant la «formule 76B »).

84. ANC, RG 13, vol. 2223, dossier 1064/1933, lettres de J. H. MacBrien, Commissioner RCMP, au ministre de la Justice Hugh Guthrie, 19 et 24 janvier 1934.

85. DCC, 29 mars 1933, p. 3491 (propos de l'hon. M. Marcil). Voir aussi ANC, RG 13, vol. 2223, dossier 1064/1933, lettre du Chief Constable Martin Bruton à A. Blackwood, Deputy-Attorney General de la Saskatchewan, 23 février 1933.

86. DCC, 29 mars 1933, pp. 3496-3500. Cette insertion, absente du projet de loi $n^{\circ} 53$ du 20 mars 1933, se retrouve dans la loi du 9 mai 1933, chapitre 25, article 1.

87. Loi du 23 juin 1936, chapitre 29, article 2. Voir aussi ANC, RG 13, vol. 2809, dossier 135931 : lettre du Colonel W. W. Poster, Chief Constable de Vancouver, au ministre de la Justice, 4 octobre 1935; lettre de J. H. MacBrien, Commissioner RCMP, au ministre de la Justice Ernest Lapointe, 9 janvier 1936.

88. Loi du 23 juin 1936, chapitre 29, article 3 : «pour s'en servir relativement à sa profession ou à son occupation ».

89. ANC, RG 13, vol. 2809, dossier 135931, lettre de J. H. MacBrien, Commissionner RCMP, au ministre de la Justice Ernest Lapointe, 9 janvier 1936.

90. Loi du $1^{\text {er }}$ juillet 1938, chapitre 44, article 2. L'article 119 (1) (b) du Code criminel est ici amendé. Quant aux instigateurs de cet amendement, voir : ANC, RG 13, vol. 2809, dossier 135931, lettre de J. H. MacBrien, Commissioner RCMP, au ministre de la Justice Ernest Lapointe, 9 janvier 1936; lettre de G. L. Jennings, Assistant Commissioner, Director Criminal Investigation, RCMP, au sous-ministre de la Justice, 14 septembre 1936.

91. Loi du $1^{\mathrm{er}}$ juillet 1938 , chapitre 44 , article 3 ( $\mathrm{La}$ « formule $76 \mathrm{E}$ » se trouve à l'article 55 ) - ANC, RG 13, vol. 2809, dossier 135931, lettre de J. H. MacBrien, Commissioner RCMP, au ministre de la Justice Ernest Lapointe, 9 janvier 1936.

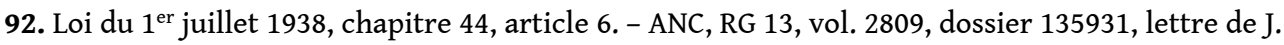
H. MacBrien, Commissioner RCMP, au ministre de la Justice Ernest Lapointe, 15 novembre 1937.

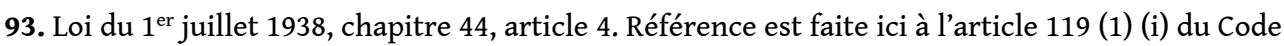
criminel. Voir aussi ANC, RG 13, vol. 2809, dossier 135931, lettre de J. H. MacBrien, Commissioner RCMP, au ministre de la Justice Ernest Lapointe, 9 janvier 1936.

94. Loi du $1^{\mathrm{er}}$ juillet 1938 , chapitre 44 , article 7.

95. Procureurs généraux de la Saskatchewan et du Nouveau-Brunswick, autorités policières (dont le Brig.-General Draper, Chief Constable de Toronto), compagnies ferroviaires, milieu torontois de l'éducation et Chambre de commerce d'Hamilton sont au rang des demandeurs de réformes. Voir : ANC, vol. 2223, dossier 1064/1933 et vol. 2809, dossier 135931. 
96. Loi du $1^{\mathrm{er}}$ juillet 1938, chapitre 44, article 9. Les raisons d'accorder le permis devant être stipulées sur la « formule $76 \mathrm{~F}$ » prévue à cet effet par l'article 55 de ladite loi, le contrôle en la matière est donc total.

97. L'alinéa (b) de l'article 119 (1) du Code dont il est ici question est amendé le $1^{\text {er }}$ juin 1950 , chapitre 11, article 2. «It has been found that this paragraph [b] is of little value and adds greatly and without compensating advantage to the work of the police. An alien will still be subject of course to all the requirements as to registration and permits that apply to citizens. The[se] requirements imposed upon citizens [...] are very strict and the additional requirements imposed upon aliens byparagraph (b) add little if anything by way of security or general law enforcement ": Bill I, As passed by the Senate, 24th March, 1950 (Explanatory Notes). Voir encore : ANC, RG 13, vol. 2745, dossier 158000-15, lettre de l'office of the Commissioner, Royal Canadian Mounted Police, au sous-ministre fédéral de la Justice, March 23, 1950. Lorsque le sénateur Hayden proposera au Sénat la deuxième lecture du projet de loi I modifiant le Code criminel, il tiendra à préciser que «l'abrogation de l'alinéa b) du paragraphe 1 de l'article 119 n'amoindrit [...] en rien le Code » (DS, 21 mars 1950, p. 119).

98. Loi du 30 juin 1951, chapitre 47, article 7 (amendement au nouvel article $127 \mathrm{du}$ Code criminel).

99. Loi du 30 juin 1951, chapitre 47, article 7 (amendement au nouvel article 124 du Code criminel). Cette réforme de l'enregistrement des armes à feu a été demandée par le commissaire de la gendarmerie royale du Canada: ANC, RG 13, vol. 2745, dossier 158000-15, lettre de S. T. Wood au ministre de la Justice Garson, 11 mai 1950.

100. Dandurand (1982, p. 250). «Lorsque cette loi de 1951 est étudiée au Parlement, elle ne rencontre guère d'opposition ", précise-t-il. Cette revision de 1951 est traitée par Dandurand (1982, pp. 246-250).

\section{RÉSUMÉS}

Le Code criminel canadien remonte à 1892. Dans le champ des armes à feu et ce jusqu'à l'aube de la Deuxième Guerre mondiale, le législateur canadien est intervenu plusieurs fois pour amender ce recueil d'interdits. Dans cet article, l'auteur analyse les fluctuations légales du Code en les mettant systématiquement en rapport avec l'évolution conjoncturelle d'un pays en train de se former et de se complexifier. Il fait ressortir que l'échafaudage législatif canadien en matière de contrôle des armes à feu est déjà bien monté à la fin des années trente mais que cette construction juridique n'est pas exempte de clauses discriminatoires envers les "étrangers » (sujets non naturalisés britanniques). L'auteur montre qu'avec en toile de fond un déferlement migratoire considérable, ce remodelage du Code criminel logé à l'enseigne du double standard est lié d'abord aux tensions nées de la Première Guerre mondiale, puis à la montée des revendications ouvrières sur les deux scènes nationale et internationale de même qu'à la crise économique des années trente.

The Canadian Criminal Code was created in 1892. Until the dawn of World War II the Canadian legislature brought many amendments to this collection of prohibitions in the field of firearms. In this article the author analyses the legal changes which were made to the Code by linking them systematically to the socio-economic evolution of a country in a formative process of development. He demonstrates that the Canadian normative structure on the subject of firearms 
control was already established in the Criminal Code at the end of the thirties, but that this legislation was not without any discriminatory clauses against «foreigners» (un-naturalized British subjects). The author shows that against the background of a huge influx of immigrants, the remodeling of the Criminal Code with its stigmatization of foreigners was provoked by tensions which were aroused during World War I, by the rise of workers movement on both the national and international scene, and by the Great Depression.

\section{AUTEUR}

\section{GÉRALD PELLETIER}

CIRCEM, Université d'Ottawa, 34 rue Stewart, Ottawa, K1N6N5, gpelleti@uottawa.ca Gérald Pelletier est chercheur au CIRCEM (Centre interdisciplinaire de recherche surla citoyenneté et les minorités) et au programme de recherche sous la direction d'Alvaro Pires, Chaire de recherche du Canada en traditions juridiques et rationalité pénale. Ses recherches portent principalement sur l'histoire des modifications apportées au Code criminel canadien entre 1892, année de son édiction, et 1970. Sur l'histoire du Code criminel canadien, il a publié : « Le Code criminel canadien 1892-1927 : Études des acteurs sociaux », The Canadian Historical Review, 1998, 79, 2, pp. 261-303 (avec A. Cellard); « La construction de l'ordre pénal au Canada, 1892-1927 : approches méthodologiques et acteurs sociaux », Déviance et Société, 1999, 23, 4, pp. 367-393 (avec A. Cellard); et «L'énigme des demandes de modifications législatives au Code criminel canadien », in Fraile P. (Éd.), Modelar para gobernar. El control de la poblacion y el territorio en Europa y Canada, Editora Universidad de Barcelona, 2001, pp. 207-231 (avec A. Pires et A. Cellard). En outre, il est notamment co-auteur du livre Histoire de l'Outaouais, Institut québécois de recherche sur la culture, 1994, 876 pages. Cet article a bénéficié d'un appui financier du Conseil de recherches en sciences humaines (projet Histoire du Code criminel canadien). Nous remercions aussi André Cellard, professeur au Département de criminologie de l'Université d'Ottawa, d'avoir relu notre article et de l'avoir enrichi de précieux commentaires. Son concours ainsi que celui de Chantal Wade ont été fort importants dans la gestation de ce texte. 